\title{
Numerical study of the transition between slow reaction and ignition in a cylindrical vessel
}

\author{
J. Melguizo-Gavilanes ${ }^{*, a, b}$, P.A. Boettcher, ${ }^{\mathrm{c}}$, R. Mével $^{\mathrm{d}, \mathrm{e}}$, J. E Shepherd ${ }^{\mathrm{a}}$ \\ ${ }^{a}$ Graduate Aerospace Laboratories, California Institute of Technology (GALCIT), Pasadena, CA, \\ USA \\ ${ }^{b}$ Institut Pprime, UPR 3346 CNRS, ISAE-ENSMA, BP 40109, 86961 Futuroscope-Chasseneuil \\ Cedex, FRA \\ ${ }^{c}$ BOEING Research and Technology, Ladson, SC, USA \\ ${ }^{d}$ Center for Combustion Energy, Tsinghua University, Beijing 100084, China \\ ${ }^{e}$ Department of Automotive Engineering, Tsinghua University, Beijing 100084, China
}

\begin{abstract}
A numerical study of a transiently (uniformly/non-uniformly) heated cylindrical reactor was performed using a computationally inexpensive one-step model capable of capturing the experimentally observed transition behavior from slow to fast reaction. The methodology used to find the kinetic parameters of the simplified model was described in detail. A parametric study using a control volume (0-D) thermal ignition model provided transition maps due to changes in heating rate, initial pressure and composition. Two-dimensional reactive Navier-Stokes equations were used to examine the fluid mechanics and chemical reaction leading to slow or fast consumption of the mixture. During uniform heating, a dynamic buoyancy flow is induced in which the mixture rises along the walls and turns at the centerline creating two well defined vortical structures. Once significant chemical heat release is generated, the flow reverses. During non-uniform heating, the flow field is composed of two large vortices in the center of the vessel, and two sets of smaller vortices trapped at the top and bottom of the reactor. Depending on the heating rate, and irrespective of the mode of heating, the mixture undergoes either slow oxidation or ignition whereby a flame that propagates from the top of the vessel consumes the mixture.
\end{abstract}

Key words: low temperature ignition, transition slow-fast reaction, industrial

\footnotetext{
*Corresponding author: josue.melguizo-gavilanes@cnrs.pprime.fr
} 
safety, simplified chemical kinetics model

\section{Introduction}

Thermal ignition of flammable gaseous mixtures from a hot surface is a major concern for a wide range of industrial activities including commercial aviation, nuclear power plants and industrial chemical processes [1, 2, 3]. Typical ignition sources include concentrated hot surfaces (glow plugs) [2, 3, 4, 5], moving hot particles $[6,7,8,9]$ and extended hot surfaces (heated vessels) [1, 10]. Minimizing the risk of accidental combustion events through updated safety regulations and improved engineering design calls for a deep understanding of this ignition phenomena.

When a combustible mixture reacts in a closed volume a thermal explosion can take place if the rate of heat production by chemistry exceeds the rate of heat loss through the vessel's walls. The thermal ignition theory of Semenov [11] and Frank-Kamenetskii [12] considered two limiting cases, respectively: (1) very efficient, instantaneous mixing so that the mixture is assumed to be spatially uniform -unsteady thermal theory; (2) no mixing/motion, allowing for temperature gradients to develop inside the reactor, hence the transport of heat occurs by conduction only -steady thermal theory. Both, Semenov and Frank-Kamenettski, found critical conditions as functions of their non-dimensional numbers. These critical values have been calculated numerically neglecting the consumption of reactants for various geometries [12].

Intermediate cases, those where the temperature gradients become sufficiently large, result in motion of the gas inside the reactor due to buoyancy. Free or natural convection can influence the progress of the chemical reaction resulting in complete suppression of the thermal runaway, or in longer ignition delay times [13]. Previous studies of the effects of natural convection on thermal explosion have considered reactors where the temperature of the wall is held constant, and systems that neglect reactant consumption $[14,15,16,17,18]$. More recent work includes an asymptotic analysis performed by Boddington et al. $[19,20]$ in which consumption of reactant 
and external heat transfer is considered in the purely diffusive limit. They found that external heat transfer has a more significant effect in spherical reactors than in infinite cylinders or parallel plates. Liu et al. [14, 21] described the explosive limits as a function of ratios of the time scales of the physical processes at play in the system, namely diffusion of heat and species, natural convection, and chemical heat release. This framework resulted in a new regime diagram in which the transition to explosion is explained in terms of non-dimensional groups that have clear physical meaning. Campbell [22], extended this work to include external heat transfer. Iglesias et al. [23] investigated thermal explosions in spherical vessels at large Rayleigh numbers with similar simplifying assumptions. They varied the Damköhler and Rayleigh numbers to discriminate between different explosion regimes under isothermal wall conditions. To date, no previous work has been concerned with analyzing in detail, from a fluid mechanical perspective, the full sequence of events leading to a thermal explosion in a cylindrical vessel when its walls are heated at a prescribed rate.

We consider the configuration used by Boettcher et al. [1] who studied the autoignition of $n$-hexane/air mixtures. A vessel containing the reactive mixture was heated externally at a finite rate. Using this setup, it was experimentally demonstrated that a flammable mixture subjected to a range of heating rates is consumed in two different ways. If the heating rate is low, a slow reaction occurs; if the heating rate is high, an ignition event takes place. The slow reaction is characterized by a slow consumption of the reactants at essentially constant temperature and pressure, while the ignition event is associated with a thermal runaway and a significant pressure increase. The mixture was observed to transition to ignition with increasing heating rate, pressure, and equivalence ratio $(0.7 \leq \phi \leq 1.4)$. This behavior was also investigated computationally using a control volume zero-dimensional (0D) model that extended the classical theory of Semenov by using a time dependent wall temperature and a detailed reaction mechanism for describing the chemistry [1]. Analyses of the chemical reaction pathways and energy balance showed that the competition between the chemical energy release rate and heat losses rate when close to 
the auto-ignition temperature can induce a composition change, so that an initially flammable mixture is turned into a non-flammable mixture. However, the large number of chemical species and reactions involved during low-temperature oxidation of hydrocarbon fuels results in very lengthy simulation times (even in 0-D) and makes it impractical to carry out comprehensive parametric studies to investigate complex industrial configurations using multidimensional numerical simulations.

The present paper is divided in two main parts: (i) the development of a computationally inexpensive one-step chemical model giving the range of kinetic parameters required to capture the transition behavior from slow to fast reaction, together with a parametric study using a control volume 0-D model to provide transition maps due to changes in heating rate, initial pressure and composition; (ii) twodimensional (2-D) simulations of the experiments carried out by Boettcher et al. [1] using the one-step model developed in (i). Rather than simply finding the boundary between explosive and non-explosive behavior as a function of initial pressure and composition like in previous analytical, experimental and numerical work (a complete list can be found in [14]), we use 2-D computations to investigate in detail the flow field and the competition that develops inside the reactor between diffusive and convective losses, and the chemical heat release as the mixture is uniformly and non-uniformly heated. To the authors' knowledge, no studies are available in the literature where the full evolution is considered from early stages of heating, ignition and flame propagation to full consumption of the mixture for reactors whose walls are heated at a prescribed rate like in the present study.

\section{Control volume zero-dimensional (0-D) model}

The thermal ignition process is described using a thermodynamic and chemical kinetic model. The thermodynamic model is given by the conservation of energy for a stationary constant volume and constant mass, $m$, system. The system exchanges energy with its surroundings through heat transfer at the wall, $Q_{w}$, which can be into or out of the system depending on the relative temperatures of the gas and the 
wall.

$$
\Delta U=Q_{r}+Q_{w}
$$

where $\Delta U$ is the change in internal energy of the system. Assuming that the gas obeys the ideal gas equation and has constant specific heat, yields

$$
m c_{v} \frac{d T}{d t}=\dot{Q}_{r}+\dot{Q}_{w}
$$

where the energy release rate due to chemical reactions, $\dot{Q}_{r}$, is in competition with the energy transfer rate at the wall, $\dot{Q}_{w}$. In Eqn. 2, $c_{v}$ is the specific heat at constant volume, and $T$ is the gas temperature. The heat transfer with the wall is expanded from the original Semenov theory to include the initial temperature of the wall, $T_{w}^{0}$, and the rate at which it is heated, $\alpha[1,11]$.

$$
\dot{Q}_{w}=S \bar{h}\left(T_{w}^{0}+\alpha t-T\right)
$$

where $S$ is the surface area and $\bar{h}$ is the heat transfer coefficient. The energy release rate is found by assuming that the reaction progresses in one irreversible step from reactant to product $(R \rightarrow P)$. The rate at which this reaction progresses depends on the temperature, $T$, and the molar concentration of reactant , $[R][24]$.

$$
\frac{d[P]}{d t}=-\frac{d[R]}{d t}=\dot{\omega}=k(T)[R]^{n}
$$

In Eq. 4, $\dot{\omega}$ is the molar production rate per unit volume, $k$ is the reaction rate, and $n$ is the effective reaction order. Using the approach from [25], we express the molar concentration using the ideal gas law, $p V=n R_{u} T$,

$$
[i]=\frac{n_{i}}{V}=\frac{p_{i}}{R_{u} T}=\frac{X_{i} p}{R_{u} T}=\frac{X_{i}}{W_{i}} \rho,
$$

where $n, p, \rho, X, W$ are the number of moles, partial pressure, density, mole fraction, and molecular weight, respectively. The variables without the subscript " $i$ " represent mixture properties. Otherwise, they represent the properties of either the reactant or the product.

The reaction is expressed as a function of a reaction progress variable, $\lambda$, which is equivalent to the mass fraction of the products, $Y_{P}$.

$$
\lambda=Y_{P}=X_{P} \frac{W_{P}}{W}
$$


Expressing Eqn. 4 in terms of mass fractions using Eqns. 5 and 6 yields

$$
\frac{d Y_{P}}{d t}=\frac{W_{P}^{2}}{W \rho} k(T)\left(\frac{X_{R}}{W_{R}} \rho\right)^{n} .
$$

The rate of progress of the chemical reaction is governed by an Arrhenius rate law $[24]$,

$$
k(T)=A \exp \left(-\frac{E_{a}}{R_{u} T}\right),
$$

where $A$ is the pre-exponential, $E_{a}$ is the activation energy and $R_{u}$ is the universal gas constant.

Substituting Eqn. 8 into Eqn. 7 and rearranging, the evolution of $Y_{P}$ becomes

$$
\frac{d Y_{P}}{d t}=\left(A \frac{W_{P}^{2}}{W} \frac{X_{R}^{n}}{W_{R}^{n}}\right) \rho^{n-1} \exp \left(-\frac{E_{a}}{R_{u} T}\right) .
$$

Restating the equation in terms of progress variable, $\lambda$, and introducing the consumption term, $(1-\lambda)$, yields

$$
\frac{d \lambda}{d t}=Z(1-\lambda) \rho^{n-1} \exp \left(-\frac{E_{a}}{R_{u} T}\right)
$$

where the terms in parenthesis in Eqn. 9 are assigned to the pre-exponential factor $Z[25]$.

Finally, the energy release rate in Eqn. 2 is the product of the energy stored in the chemical mixture, $Q_{c}$, and the rate at which this energy is released, $d \lambda / d t$,

$$
\dot{Q}_{r}=Q_{c} \frac{d \lambda}{d t}=m q_{c} \frac{d \lambda}{d t}=m q_{c} Z(1-\lambda) \rho^{n-1} \exp \left(-\frac{E_{a}}{R_{u} T}\right),
$$

where $q_{c}$ is the chemical energy content per unit mass of reactant $R$.

The complete coupled equations describing this thermal ignition model are

$$
\begin{gathered}
\frac{d T}{d t}=\frac{q_{c}}{c_{v}} Z(1-\lambda) \rho^{n-1} \exp \left(-\frac{E_{a}}{R_{u} T}\right)+\frac{S \bar{h}}{m c_{v}}\left(T_{w}^{0}+\alpha t-T\right), \\
\frac{d \lambda}{d t}=Z(1-\lambda) \rho^{n-1} \exp \left(-\frac{E_{a}}{R_{u} T}\right) .
\end{gathered}
$$

\section{Calculation of model parameters}

In the current thermal ignition model a number of parameters are fixed by the physical system used in the experimental study [1], such as the surface area and 
volume (see Table 1). Other parameters, such as the activation energy and chemical energy content depend on the type of mixture used, and are determined with the use of a detailed chemical model and the available experimental data.

Table 1: One-step model parameters.

\begin{tabular}{llll} 
Parameter & Value & Units & Description \\
\hline \hline$V$ & $4.27 \times 10^{-4}$ & $\mathrm{~m}^{3}$ & volume \\
$S$ & $3.36 \times 10^{-2}$ & $\mathrm{~m}^{2}$ & surface area \\
$\bar{h}$ & 10 & $\mathrm{~W} \mathrm{~m}^{-2} \mathrm{~K}^{-1}$ & heat transfer coefficient \\
$c_{v}$ & 980 & $\mathrm{~J} \mathrm{~kg}^{-1} \mathrm{~K}^{-1}$ & specific heat at constant volume \\
$R_{u}$ & 8.314 & $\mathrm{~J} \mathrm{~mol}^{-1} \mathrm{~K}^{-1}$ & universal gas constant \\
$E_{a}$ & $1.48 \times 10^{5}$ & $\mathrm{~J} \mathrm{~mol}^{-1}$ & activation energy \\
$q_{c}$ & $2.461 \times 10^{6}$ & $\mathrm{~J} \mathrm{~kg}^{-1}$ & chemical energy content \\
\hline \hline
\end{tabular}

\subsection{Heat transfer}

The average heat transfer coefficient, $\bar{h}$, was determined by performing an inert two-dimensional simulation of the buoyancy flow induced by the heating of the reactor's wall (see section 5 for details) to confirm typical values for natural convection of gases $\left(2-25 \mathrm{~K} / \mathrm{m}^{2} \mathrm{~K}\right)$ given in heat transfer textbooks [26]. Using the standard definition, $\bar{h}=q^{\prime \prime} /\left(T_{w}(t)-T\right)$, where $q^{\prime \prime}$ is the wall heat flux, and the difference $\left(T_{w}(t)-T\right)$ is the time dependent thermal gradient, with $T_{w}(t)=T_{w}^{0}+\alpha t$. The average heat transfer coefficient was found to be $10 \mathrm{~W} / \mathrm{m}^{2} \mathrm{~K}$

\subsection{Thermo-chemical parameters}

In order to include realistic thermodynamic and chemical parameters in the onestep reaction model, 0-D calculations were carried out using a detailed reaction mechanism, namely the updated Ramirez et al. model [27, 28]. It includes 531 chemical species and 2,628 reactions. A detailed validation of the model of Ramirez

\footnotetext{
${ }^{1}$ for a slightly lean $(\Phi=0.9) n$-hexane mixture at $p_{o}=101.3 \mathrm{kPa}$.
} 
was performed in our previous study on the effect of heating rate on $n$-hexane oxidation, see [1].

\subsubsection{Energy content}

The specific energy of the mixture, $q_{c}$, is calculated from the detailed mechanism assuming an adiabatic system where all of the stored chemical energy is used to heat the product species until chemical equilibrium is reached. The energy content of the system can then be extracted by cooling the system back to its initial temperature and computing the difference in internal energy. Calculations were performed using Cantera [29] for $\phi=0.4-3, T_{o}=298 \mathrm{~K}$, and $p_{o}=20.3-202.6 \mathrm{kPa}$ as shown in Fig. 1. Only minor changes were observed relative to previous calculations [30].

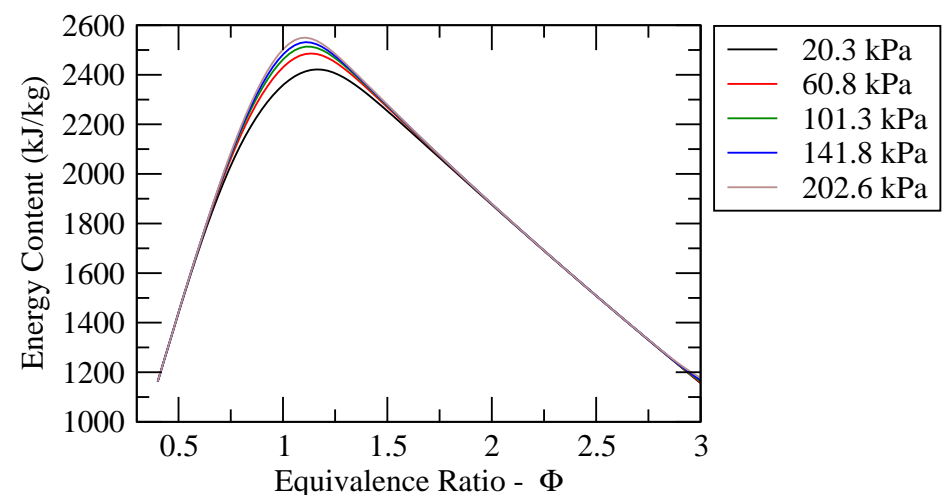

Figure 1: Control volume (0-D) model - evolution of energy content per unit of mass as a function of equivalence ratio. Conditions: $T_{o}=298 \mathrm{~K}$ and $p_{o}=20.3-202.6 \mathrm{kPa}$.

\subsubsection{Activation energy}

Ignition delay-time calculations were performed assuming a constant volume reactor to obtain the activation energy, $E_{a}$, as a function of pressure and equivalence ratio. The ignition delay-time was defined as the time to maximum pressuregradient. Computations were carried out over a period of $2000 \mathrm{~s}$ for $\phi=0.4-3$, $p_{o}=20.3-202.6 \mathrm{kPa}$, and $T_{o}=500-1100 \mathrm{~K}$. Previous work $[2,30]$ shows how the activation energy is calculated by fitting the low temperature ignition delay time and calculating the slope of this curve. The changes in activation energy with initial pressure and equivalence ratio were negligible yielding an approximately constant value of $E_{a}=148,000 \mathrm{~J} / \mathrm{mol}$. 


\subsubsection{Pre-exponential Factor}

The pre-exponential factor, $Z$, is calibrated so that the one-step model reproduces the transition from slow reaction to ignition observed in the 0-D thermal ignition model using detailed chemistry [30]. For a stoichiometric mixture at $101 \mathrm{kPa}$ the transition occurred when the heating rate was increased from $10.75 \mathrm{~K} / \mathrm{min}$ to $10.80 \mathrm{~K} / \mathrm{min}$. This transition behavior is shown for the one-step model in Fig. 2. Figure 2 (Left) shows the very slight temperature increase above the heating rate for the slow reaction case as well as the large temperature increase for the ignition case. Similarly, the reaction progress variable shows a steep increase at the point of ignition in Fig. 2 (Right). The pre-exponential factors are individually fitted for two different effective reaction orders, $n=1$, and $n=2$, and determined to be $Z=8.75 \times 10^{10}$ and $Z=7.25 \times 10^{10}$, respectively.
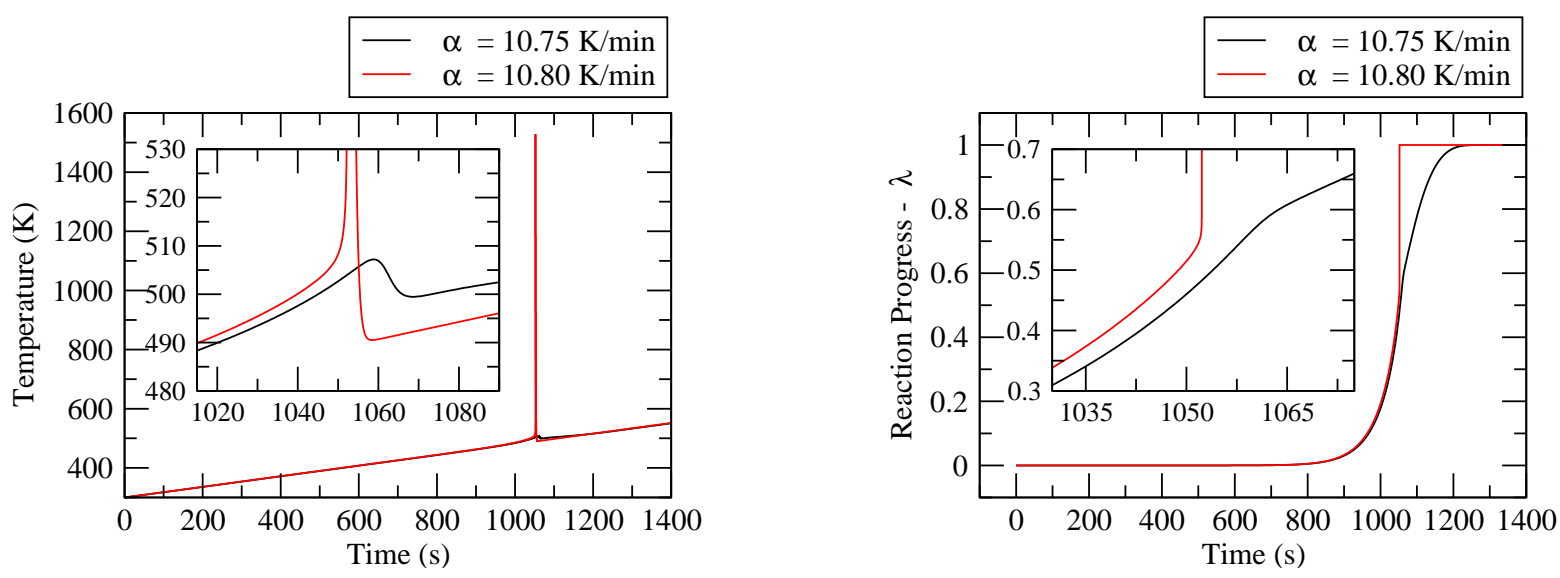

Figure 2: Control volume (0-D) model simulation results for $n=1, p_{o}=101 \mathrm{kPa}$, and $\phi=$ 1.0 showing the temperature evolution (Left) and progress variable evolution (Right) for a slow reaction and an ignition case with a small heating rate increase of $0.05 \mathrm{~K} / \mathrm{min}$.

\section{Parametric study in $0-\mathrm{D}$ - constant volume reactor}

With the established one-step model, the behavior of the gas mixture can be investigated as a function of the initial pressure, initial composition, heating rate, and reaction order. Solutions to the thermal ignition model were obtained for a pressure 
range of $p_{o}=20.3-202.6 \mathrm{kPa}$ with the equivalence ratio varying from $\phi=0.5-2.3$. The heating rate was varied from $\alpha=1 \mathrm{~K} / \mathrm{min}$ to $30 \mathrm{~K} / \mathrm{min}$ to investigate the transition behavior. An ignition case is distinguished from a slow reaction case by the temperature overshoot, $\Delta T$, above that prescribed by the heating rate. The transition is marked by a threshold temperature overshoot of $50 \mathrm{~K}$. Figure 3 (Left) shows the combined transition map for a unity reaction order $(n=1)$. Each line represents the transition curve above which the mixture ignites and below which the mixture undergoes a slow reaction. Each curve shows a minimum required heating rate near stoichiometric composition consistent with the energy content reaching a maximum (see Fig. 1). As the pressure is increased, the ignition regime widens away from near-stoichiometric mixtures monotonically; at a fixed equivalence ratio the heating rate required for ignition decreases with increasing pressure.

For a unity reaction order, the energy balance, Eqn. 12, readily shows the direct influence of the energy content, heating rate, and initial pressure (through changing the mass). Increasing the energy content increases the energy release; increasing the heating rate decreases the heat loss; and increasing the initial pressure increases the mass while not affecting the surface area and heat transfer coefficient hence decreasing the heat loss. All of these trends are seen in Fig. 3 (Left).

When the effective reaction order is changed to $n=2$, the pressure also affects the energy release rate and the reaction progress. The results given in Fig. 3 (Right) show that same monotonic trend is still observed, but the individual transition curves are shifted. Changing the effective reaction order from 1 to 2 shifts the transition curve below atmospheric pressures to higher heating rates. At elevated pressures, this trend is reversed and an increase in effective reaction order from 1 to 2 decreases the required heating rate. The dashed lines in Fig. $3(n=1)$ make this trend visible. The atmospheric pressure curve is unaffected by the change in reaction order as is the pivot about which the curves shift. 

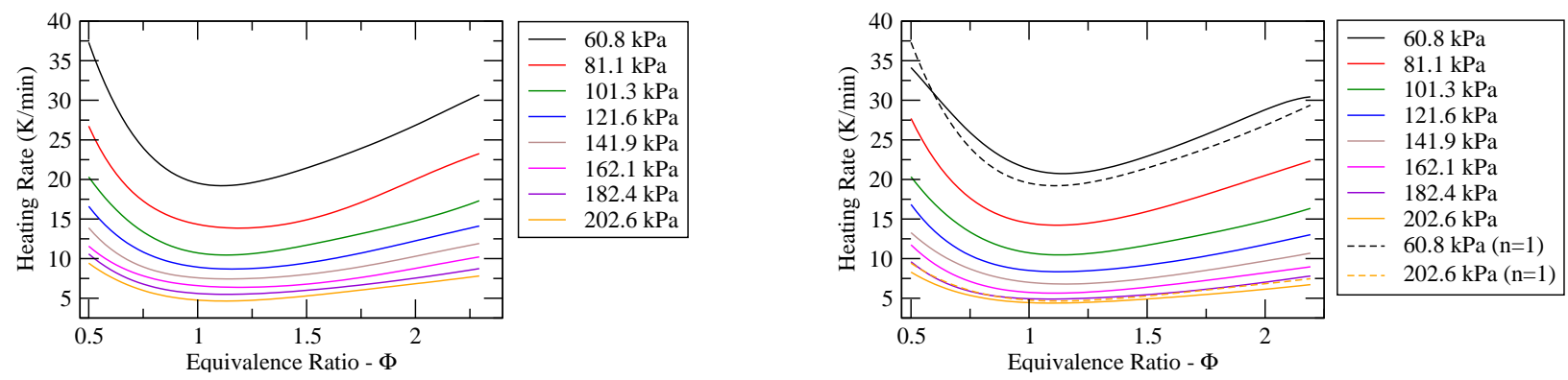

Figure 3: Control volume (0-D) model simulation results - plots of the transition curves for reaction order $n=1$ (Left) and $n=2$ (Right). Ignition occurs for initial conditions above the transition curve and a slow reaction occurs below.

The thermal ignition model just described succeeded at capturing the transition from slow to fast reaction by assuming that the mixture inside the vessel is perfectly mixed hence neglecting spatial temperature gradients in the reactor. Evidently, these gradients are unavoidably present in reality and are responsible for inducing a buoyant flow inside the vessel that could delay or suppress ignition. The next sections examine the fluid mechanics and chemical reaction leading to slow and fast consumption of the mixture through 2-D simulations.

\section{Multidimensional Simulations: physical model, numerical approach and model parameters}

The flow and ignition inside the cylindrical vessel is governed by the variable density, reactive Navier-Stokes equations with temperature dependent transport properties:

$$
\begin{gathered}
\frac{\partial \rho}{\partial t}+\nabla \cdot(\rho \mathbf{u})=0, \\
\frac{\partial(\rho \mathbf{u})}{\partial t}+\nabla \cdot(\rho \mathbf{u u})=-\nabla p+\nabla \cdot \tau+\rho \mathbf{g}, \\
\frac{\partial\left(\rho h_{s}\right)}{\partial t}+\nabla \cdot\left(\rho \mathbf{u} h_{s}\right)=\nabla \cdot\left(\kappa / c_{p} \nabla h_{s}\right)+q_{c} \dot{\Omega}_{R}, \\
\text { with } p=\rho \bar{R} T, \quad \tau=\mu\left[\nabla \mathbf{u}+(\nabla \mathbf{u})^{T}\right]-\frac{2}{3} \mu(\nabla \cdot \mathbf{u}) \mathbf{I} .
\end{gathered}
$$

The Sutherland Law [31], the Eucken Relation [32] and the JANAF polynomials [33] are used to account for the functional temperature dependence of mixture viscosity 
$(\mu)$, thermal conductivity $(\kappa)$ and specific heat $\left(c_{p}\right)$ respectively. The chemistry is modeled using an irreversible one-step scheme $(R \rightarrow P)$ as described in section 3 . Using subscript $R$ for reactants, species mass conservation can be written as:

$$
\frac{\partial\left(\rho Y_{R}\right)}{\partial t}+\nabla \cdot\left(\rho \mathbf{u} Y_{R}\right)=\nabla \cdot\left(\rho D_{R} \nabla Y_{R}\right)-\dot{\Omega}_{R} \text { with } \dot{\Omega}_{R}=\rho Y_{R} Z \exp \left(-E_{a} / R_{u} T\right)
$$

where $\rho$ is density, $\mathbf{u}$ is the velocity vector, $p$ is pressure, $h_{s}$ is the mixture sensible enthalpy, $\mathbf{g}$ is the gravitational acceleration, $q_{c}$ is the stored chemical energy, $\mathbf{I}$ is the identity matrix, $Y_{R}$ is the mass fraction of reactants, $Z$ is the pre-exponential factor, $E_{a}$ is the activation energy, and $R_{u}$ and $\bar{R}$ are the universal and specific gas constants, respectively. The Lewis number is assumed to be unity which results in $\kappa / c_{p}=\rho D_{R}$. The thermal diffusivity of reactants is used to model its mass diffusivity.

The equations above are integrated in two dimensions using the Open source Field Operation And Manipulation (OpenFOAM) toolbox [34]. The spatial and temporal discretization details along with the iterative techniques used to solve the linear systems that result from discretizing the governing equations can be found in [8]. The OpenFOAM framework has been used successfully for a variety of studies including direct numerical simulation of turbulent premixed flames [38, 39], chemical kinetic studies of turbulent non-premixed jet flames [40], laminar coflow flames [41, 42], flows with surface reactions [43], fuel spray mixing [44, 45] and thermal ignition $[4,5,6,7,8,9]$.

The geometry simulated corresponds to a vertical cross section of the cylindrical reactor used in [2] (5 cm in diameter and $22 \mathrm{~cm}$ in length). Figures 4 and 5 show a schematic of the experimental setup and the computational mesh -full O-grid with square center piece to avoid singularities and small angles at high resolutions. There are approximately 10,000 points in the computational domain, compressed near the wall of the reactor, with a minimum cell size of $60 \mu \mathrm{m}$ normal to the wall to allow for enough resolution to resolve the thermal and hydrodynamic boundary layers. 


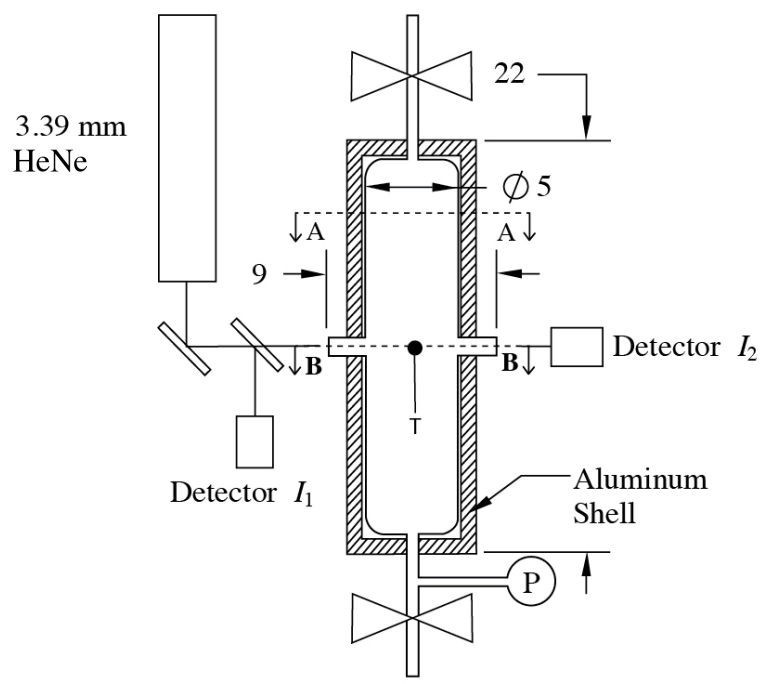

A - A : Uniform heating

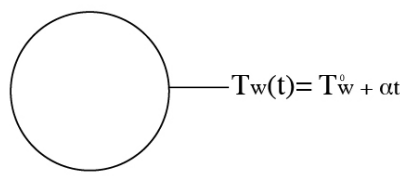

B - B : Non - uniform heating

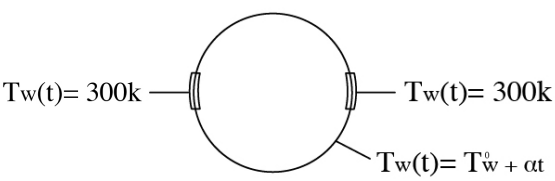

Figure 4: Schematic of experimental setup used in [2] (top view), and corresponding vertical cross sections for simulation of uniform and non-uniform heating.

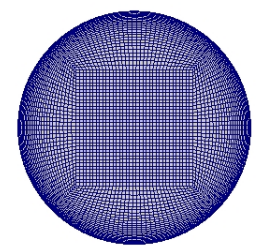

Figure 5: Computational domain for cylindrical reactor - O-grid with square centerpiece and cell compression near walls.

Initial conditions are $p_{o}=101 \mathrm{kPa}, T_{o}=T_{w}^{0}=300 \mathrm{~K}, \mathbf{u}_{o}=(0,0) \mathrm{m} / \mathrm{s}$, and $Y_{R}^{0}=1$. No-slip condition and a prescribed temperature ramp on the vessel's wall given by $T_{w}(t)=T_{w}^{0}+\alpha t$ where $\alpha$ is the heating rate, are specified as boundary conditions for velocity and temperature respectively. There is no flux of species at the wall. Hence, the effects of any surface reactions have been ignored. The chemical kinetics parameters used for modeling a slightly lean $(\Phi=0.9) n$-hexane-air mixture are $q_{c}=2.461 \times 10^{6} \mathrm{~J} / \mathrm{kg}$ (see Fig. 1 ), $E_{a}=148,000 \mathrm{~J} / \mathrm{mol}$, and $Z=8.75 \times 10^{10} \mathrm{~s}^{-1}$. 


\section{Theoretical considerations: flow regime and time scales}

Before showing the 2-D simulation results is instructive to mention a few words about the flow regime in which our proposed computations lie. More specifically, testing if simple estimates based on scalings of the governing equations provide some insight. In contrast with previous work [14, 21, 22] where the onset of buoyancy was due to the temperature differences created inside the vessel as a result of heat deposition by the chemistry, here, the buoyant motion is initially induced by the heating rate imposed on the vessel's wall. Assuming that up to ignition density variations (i.e. temperature differences, $\Delta T$ ) in the vessel are small compared to the wall temperature $\left(\Delta T / T_{w}(t) \ll 1\right)$ the Boussinesq approximation applies. A scaling for velocity due to natural convection can then be found by balancing the convective and buoyancy terms in the momentum equation 15. Casting the buoyancy term, $\rho \mathbf{g}$, using the aforementioned approximation yields: $\left[\left(\rho-\rho_{o}\right) / \rho_{o}\right] \mathbf{g}$ where $\rho_{o}$ is a reference density. In the Boussinesq approximation density variations are assumed to have a fixed part and another that has a linear dependence with temperature, $\rho=\rho_{o}(1-\beta \Delta T)$, where $\beta=1 / T_{o}$ is the coefficient of thermal expansion for ideal gases and $T_{o}$ is a reference temperature. Using the previous definitions the following scaling for velocity is obtained:

$$
U \sim(L \mathbf{g} \beta \Delta T)^{1 / 2}
$$

where $L$ is a characteristic length scale (i.e. the vessel's diameter $D$ in our case). An appropriate estimate for $\Delta T$ (the temperature difference that drives the convective motion) can be found by using the heating rate imposed on the wall, $\alpha$, and a measure for the transit/turn over time (i.e. a convective time scale) of one fluid parcel to travel from the bottom of the vessel, rise along the wall and descend back to its starting point: $\Delta T \sim K \alpha D / U$ with $K=(2+\pi) / 2$. Replacing this expression in the scaling found for $U$ yields:

$$
\Delta T=\alpha^{2 / 3}\left(\frac{D}{\mathbf{g} \beta}\right)^{1 / 3} K^{2 / 3}
$$

This expression elucidates the effect of the heating rate and size of the vessel on $\Delta T$, and ultimately on the strength of the convective motion induced. A higher heating 
rate and a bigger vessel are conducive to generating larger temperature differences. On the other hand, a larger gravitational acceleration and coefficient of thermal expansion have an inverse effect on $\Delta T$.

Based on the results obtained using the thermal model discussed in Section 3, we can choose a reference temperature $T_{o}$ for $\beta$, and a heating rate value, $\alpha$, to compute the expected temperature difference, $\Delta T$, inside the vessel. Figure 2 (Left) indicated that $T_{o}=500 \mathrm{~K}$ is a temperature value close to the temperature range in which chemical activity becomes important. Similarly, Figure 3 (Left) shows that for $\Phi=0.9$ and $p_{o}=100 \mathrm{kPa}$ a value of $\alpha=15 \mathrm{~K} / \mathrm{min}$ lies inside the ignition region in the transition map shown in this figure. Using these values together with our geometrical constraint, $D=0.05 \mathrm{~m}$, and $\mathbf{g}=9.81 \mathrm{~m} / \mathrm{s}^{2}$ we obtain a $\Delta T=1.02 \mathrm{~K}$. For $\alpha=10 \mathrm{~K} / \mathrm{min}$, a value of $\alpha$ that lies in the no ignition region of the transition map shown in Fig. 3 (Left), yields a $\Delta T=0.78 \mathrm{~K}$. Temperature differences in the range of $0.7 \mathrm{~K} \leq \Delta T \leq 1.1 \mathrm{~K}$ result in flow velocities induced by buoyancy of 0.0316 $\mathrm{m} / \mathrm{s}$ and $0.0276 \mathrm{~m} / \mathrm{s}$ for $\alpha=15 \mathrm{~K} / \mathrm{min}$ and $10 \mathrm{~K} / \mathrm{min}$, respectively.
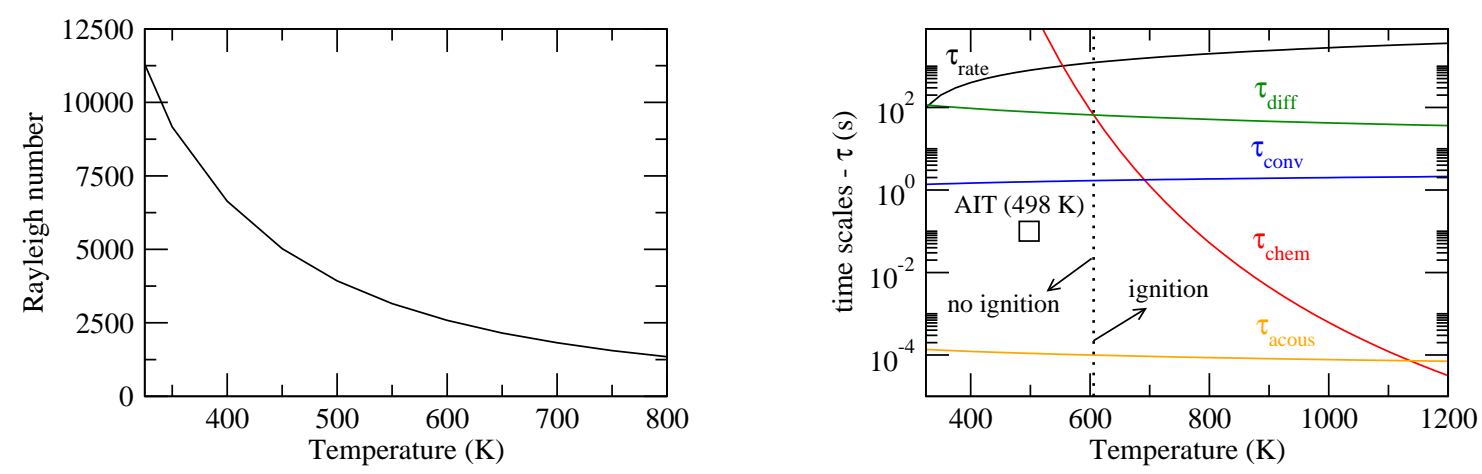

Figure 6: Estimated Rayleigh numbers (left), and time scales for heating, convection, diffusion, and chemistry as a function of temperature (right) for $15 \mathrm{~K} / \mathrm{min}$.

Since $\Delta T$ is small, we can use $T_{w}(t)$ to approximate the gas temperature inside the vessel and compute a temperature dependent Rayleigh number using $R a=$ $\mathbf{g} \beta \Delta T D^{3} / \nu \bar{\alpha}$. Due to the increase in kinematic viscosity, $\nu$, and thermal diffusivity, $\bar{\alpha}$, with increasing temperature, $R a$ decreases. See Fig. 6 (Left). Flow regimes based 
on $R a$ are divided as follows: for $R a<500$ heat transfer is controlled by conduction; for $500<R a<10^{6}$ laminar convection dominates heat transfer; and for $R a>10^{6}$ the flow is turbulent [14]. Based on the $R a$ values shown in Fig. 6 (Left), from initial stages of heating to ignition laminar convection controls the heat transfer inside the vessel.

To close the discussion is also instructive to analyze the time scales present in thermal ignition problems to shed light on the dominant physics:

$$
\begin{gathered}
\tau_{\text {rate }}=\left(T_{w}(t)-T_{w}^{0}\right) / \alpha, \tau_{\text {conv }}=D / U, \tau_{\text {diff }}=D^{2} / \bar{\alpha}, \tau_{\text {acous }}=D / \sqrt{\gamma \bar{R} T_{w}(t)} \\
\tau_{\text {chem }}=1 / Z \exp \left(-E_{a} / R_{u} T_{w}(t)\right)
\end{gathered}
$$

where $\tau_{\text {rate }}$ is the time scale associated with the heating rate, and $\tau_{\text {conv }}, \tau_{\text {diff }}, \tau_{\text {acous }}$ and $\tau_{\text {chem }}$ are the convective, diffusive, acoustic and chemical time scales, respectively, all of them temperature dependent; $\gamma=c_{p} / c_{v}$ is the ratio of specific heats.

Figure 6 (Right) shows these time scales as a function of temperature for $\alpha=$ $15 \mathrm{~K} / \mathrm{min}$. Note that $\tau_{\text {conv }}$ defined here differs from that used to calculate $\Delta T$ by a factor of $K$, and will cause the blue line shown in this plot to shift upward. $\tau_{\text {acous }}$ is significantly shorter than the rest of the time scales up to $T \sim 1000 \mathrm{~K}$. However, if heat is deposited in the gas at a time scale comparable to $\tau_{\text {acous }}$, by for instance increasing the current heating rate by four/five orders of magnitude, convective motion is not induced and pressure waves will be generated in the vessel instead; the mixture will be ignited homogeneously along the circumference of the vessel, resulting in particularly interesting pressure wave-flame interactions as the mixture is consumed from the walls towards the center of the reactor. Clarke et al. [46, 47] studied the generation of weak and strong shock waves in a confined inert gas due to rapid heat addition at the boundaries. The average convective, diffusive and acoustic time scales across the temperature range plotted are $\tau_{\text {conv }}=1.78 \mathrm{~s}, \tau_{\text {diff }}=62.54 \mathrm{~s}$ and $\tau_{\text {acous }}=9.5 \times 10^{-5} \mathrm{~s}$, respectively; $\tau_{\text {chem }}$ is by definition strongly dependent on temperature as can be seen in Fig. 6 (Right). The balancing scales up to ignition for the heating rates considered here are the diffusive and chemical time scales: for $T<$ $600 \mathrm{~K}, \tau_{\text {diff }}<\tau_{\text {chem }}$ which means that the rate of heat deposition by the chemistry is 
small compared to the rate at which heat is diffused away; for $T>600 \mathrm{~K}, \tau_{\text {chem }}<\tau_{\text {diff }}$ the opposite holds; when $\tau_{\text {chem }} \sim \tau_{\text {diff }}(T \sim 600 \mathrm{~K})$ marks the boundary between ignition and no ignition (dotted line). The autoignition temperature (AIT) reported for $n$-hexane $(498 \mathrm{~K})$ is also shown as a square symbol for reference. This simple theoretical analysis provides a good estimate of the temperature at which ignition takes place, as well as the time to ignition since a direct correlation exists between the wall temperature, $T_{w}(t)$, and the gas temperature through $\alpha$ because $\Delta T$ is small.

\section{Reference case: no buoyancy}

To better understand how buoyancy affects the ignition evolution, a case in which natural convection is neglected is considered first. The temporal evolution of the temperature of the gas, the flow structure inside the reactor, and the contributions of each of the terms in the energy equation to the ignition process are studied.

\subsection{Time evolution - ignition event}

The temperature maximum in the computational domain and reactor wall temperature are monitored during the course of the numerical integration to accurately determine the time to ignition (see Fig. 7). The main plot shows the evolution for the entire process, and the inset a close-up to the ignition event. Significant chemical activity starts shortly after the wall temperature reaches $500 \mathrm{~K}$. Ignition occurs after $\mathrm{t} \sim 1051.2 \mathrm{~s}$, the mixture is rapidly consumed in a few milliseconds, and the system relaxes to $T_{w}(t)$ after $6 \mathrm{~s}$.

\subsection{Flow analysis $-\alpha=15 \mathrm{~K} / \mathrm{min}$}

In the absence of natural convection all the important features are confined to the center of the reactor. All fields are scaled using their maximum value attained during the entire evolution described. Temperature and product mass fractions peak at this location. As time evolves, the gradients grow larger evidenced by the circular regions shrinking in both fields. The magnitude of velocity evolves differently. No motion is induced in the reactor at early times (up to $1050 \mathrm{~s}$ ). Once the temperature/density difference between the walls and the center of the reactor is large enough, flow 


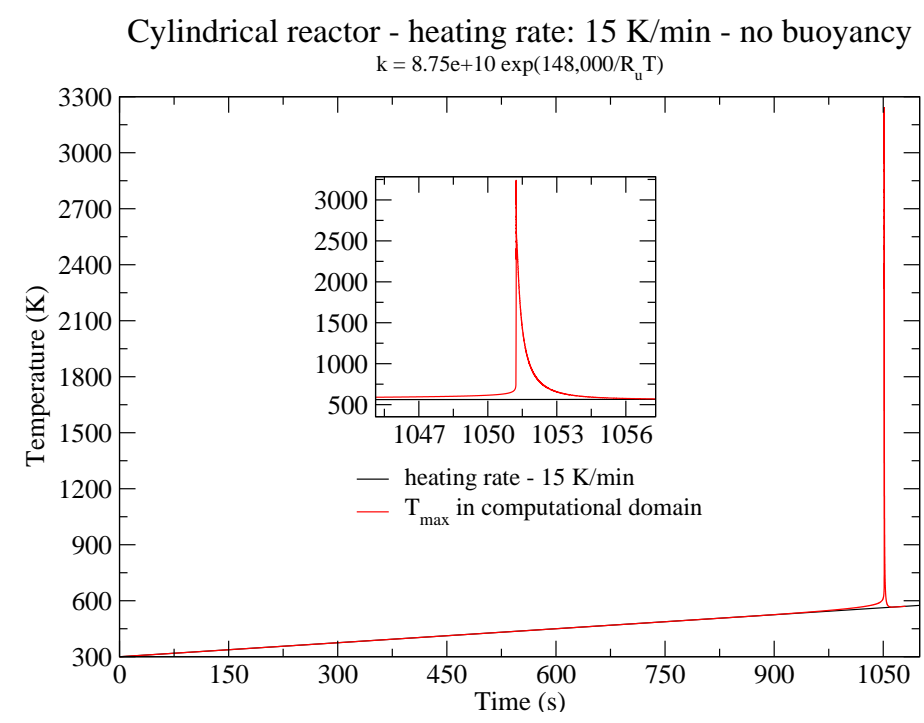

Figure 7: 2-D model simulation results - temperature histories of a uniformly heated cylindrical reactor for $\alpha=15 \mathrm{~K} / \mathrm{min}$ neglecting buoyancy.

inside the vessel starts. The gas moves radially outwards. After ignition, a flame that emanates from the center of the reactor consumes the mixture rapidly (not shown).

\subsection{Evolution of temperature, velocity and product mass fraction along vertical cen-} terline

The evolution of temperature, velocity and product mass fraction along the vertical centerline of the vessel are plotted in Fig. 9. To make the flow features visible at all times, the temperature and product mass fraction are normalized by the time dependent wall temperature, $T / T_{w}(t)$, and product mass fraction at the bottom of the vessel, $\Lambda=P / P(t)_{@ 5 c m}$, respectively. The vertical velocity, $U_{y}$, is kept in its dimensional form. Distance is measured taking the top of the vessel as a reference, and velocity is taken as positive upwards. The vertical dashed line in the plots is a visual indicator showing the center of the reactor. As mentioned above, the ignition zone is confined to the center. The growth of the chemical activity starts at $\mathrm{t}=$ 1050 s with values of $T / T_{w}(t)$ and $\Lambda$ greater than unity. From then on, the exponential dependence of the reaction rate takes over, and the ignition center grows rapidly. The vertical velocity exhibits a more dynamic evolution with appreciable 


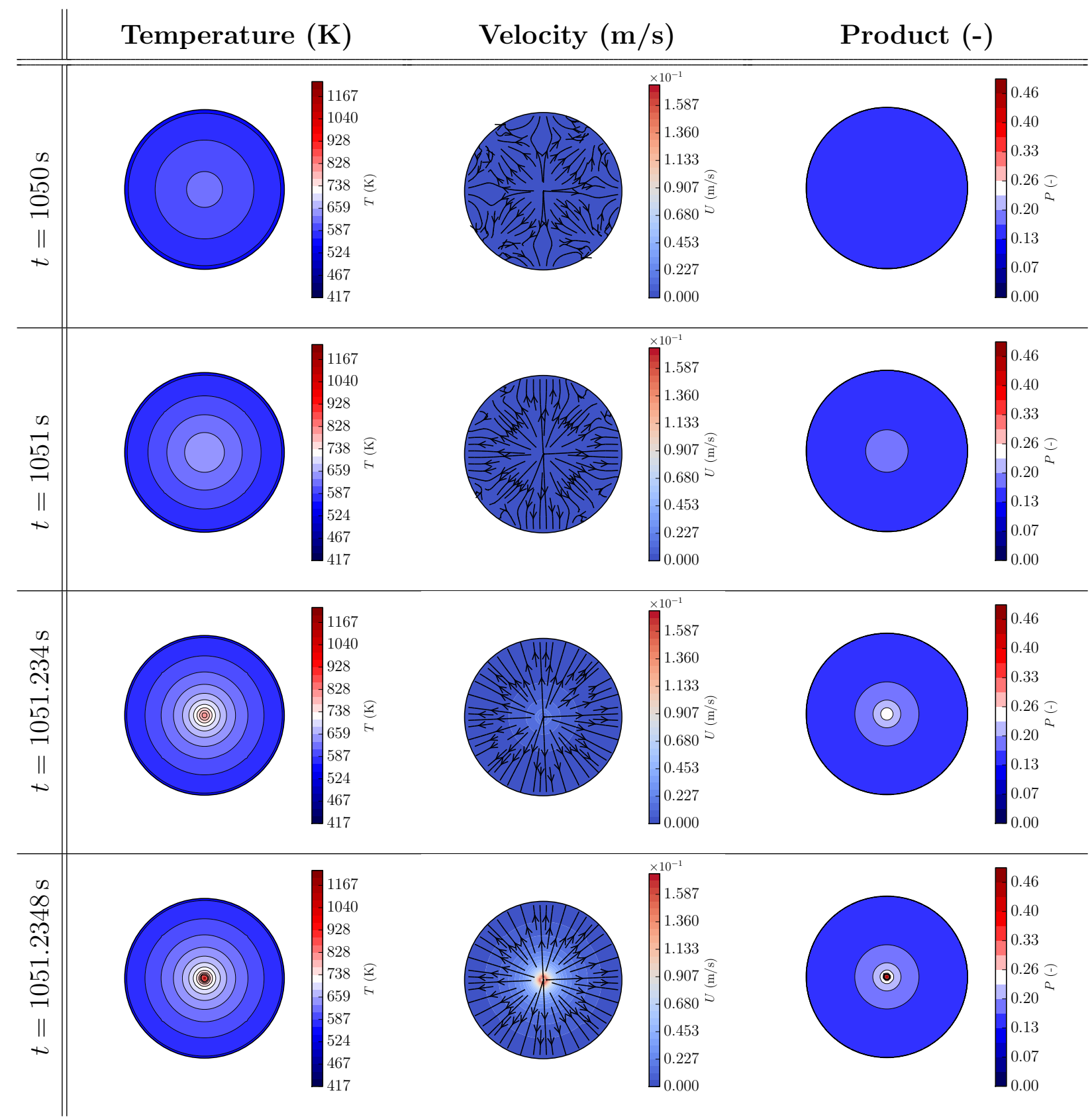

Figure 8: 2-D model simulation results - temperature (left), velocity magnitude (middle) and product mass fraction (right) fields - ignition evolution for $\alpha=15 \mathrm{~K} / \mathrm{min}$ applied to a uniformly heated cylindrical reactor neglecting buoyancy.

flow induced from the center of the vessel at $1051.23 \mathrm{~s}$. The abrupt acceleration of the gas from 1051.2344 - $1051.2348 \mathrm{~s}$ signals the birth of an ignition center. 

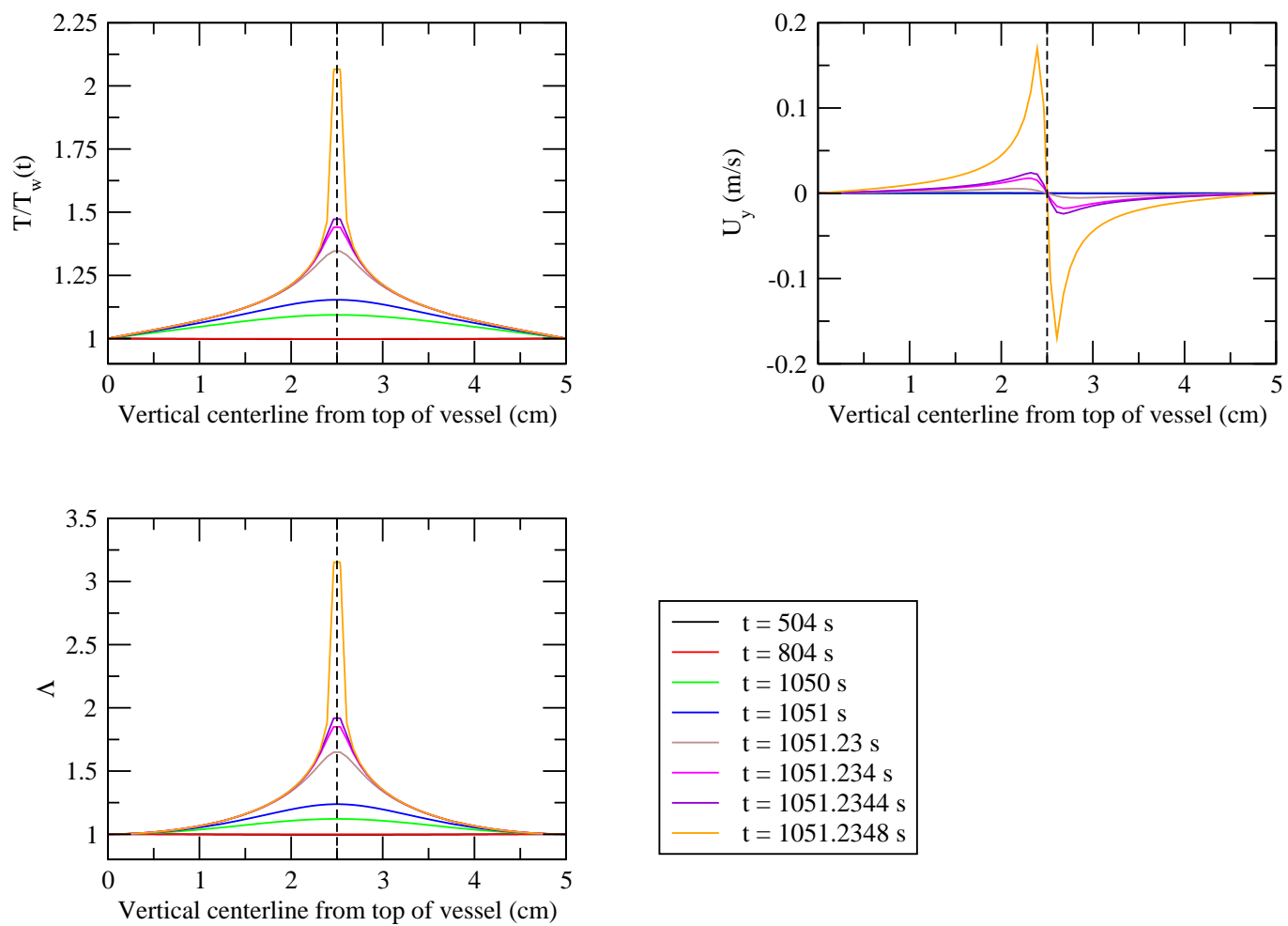

Figure 9: 2-D model simulation results - normalized temperature $\left(\mathrm{T} / \mathrm{T}_{w}(\mathrm{t})\right)$, vertical velocity $\left(\mathrm{U}_{y}\right)$ and normalized product mass fraction profiles $(\Lambda)$ along vertical centerline of heated vessel during ignition evolution for $\alpha=15 \mathrm{~K} / \mathrm{min}$ applied to a uniformly heated cylindrical reactor neglecting buoyancy.

\subsection{Energy equation analysis}

To achieve additional understanding of the physics taking place inside the reactor, the contributions of each of the terms in the energy equation and temperature are plotted along its vertical centerline (diameter), see Fig. 10 . The solid lines are the convective and diffusive heat losses, and the chemical source term given respectively by $h_{\text {Convection }}=-\nabla \cdot\left(\rho \mathbf{u} h_{s}\right), h_{\text {Diffusion }}=\nabla \cdot\left(\kappa / c_{p} \nabla h_{s}\right)$, and $h_{\text {Source }}=q_{c} \dot{\Omega}_{R}$. The dashed line is the sum of the previous terms, and the dashed-dotted line is the temperature. The plots are taken at the times shown in the two-dimensional fields to allow for a direct comparison, and show the evolution of the ignition center. Gradients are small at early times $(\mathrm{t}=1050 \mathrm{~s})$. The source term is mostly balanced by diffusion across the entire vertical centerline of the vessel. Convection 
is most active closer to the center of the reactor because incipient flow is being induced by the density differences caused by the heat release. As time progresses $(\mathrm{t}=1051-1051.234 \mathrm{~s})$, and heat deposition becomes stronger, the gradients grow larger and more flow is induced as a result. The convection term is now stronger than diffusion but their combined magnitude is not large enough to counteract the effect of the chemistry between 2-3 $\mathrm{cm}$. Ignition of the mixture occurs $0.8 \mathrm{~ms}$ later at $\mathrm{t}=1051.2348 \mathrm{~s}$.

Next, cases corresponding to two different locations along the cylinder axis are considered (see Fig 4). First, uniform heating of walls corresponding to a vertical cross section taken far away from the optical access ports, and second, non-uniform heating, which corresponds to taking the vertical cut at the windows location. The same methodology used for the reference case is applied to the analysis of both cases.

\section{Uniform heating}

\subsection{Time evolution - slow reaction and ignition event}

The heating rate was increased at $3 \mathrm{~K} / \mathrm{min}$ intervals starting from $9 \mathrm{~K} / \mathrm{min}$. A drastic change in the reaction behavior occured when the rate was increased from 12 to $15 \mathrm{~K} / \mathrm{min}$. From initially having slow consumption of the mixture at $12 \mathrm{~K} / \mathrm{min}$ to an ignition event at $15 \mathrm{~K} / \mathrm{min}$. Note that the transition took place at a slightly higher heating rate than the one predicted by the thermal model described in Section 4, Fig. 3 (Left). Figure 11 shows the temporal evolution of the maximum temperature in the computational domain and reactor wall temperature. The insets show in detail the two behaviors observed which are very similar in nature, as expected, to those shown in Fig. 2 (Left). In both cases, a weak departure from the temperature ramp imposed at the wall occurs around $500 \mathrm{~K}$ due to the onset of chemical energy release. For the $12 \mathrm{~K} / \mathrm{min}$ case, chemical activity starts after $1125 \mathrm{~s}$ of heating, reaction proceeds very slowly to completion over $200 \mathrm{~s}$ and returns to $T_{w}(t)$ after $\sim 400 \mathrm{~s}$. The maximum temperature difference between the reactor's wall temperature and maximum inside of the vessel at the peak of reaction is $33 \mathrm{~K}$ and occurs at $1404 \mathrm{~s}$. For the $15 \mathrm{~K} / \mathrm{min}$ case, a very different evolution is observed. Chemical activity starts 

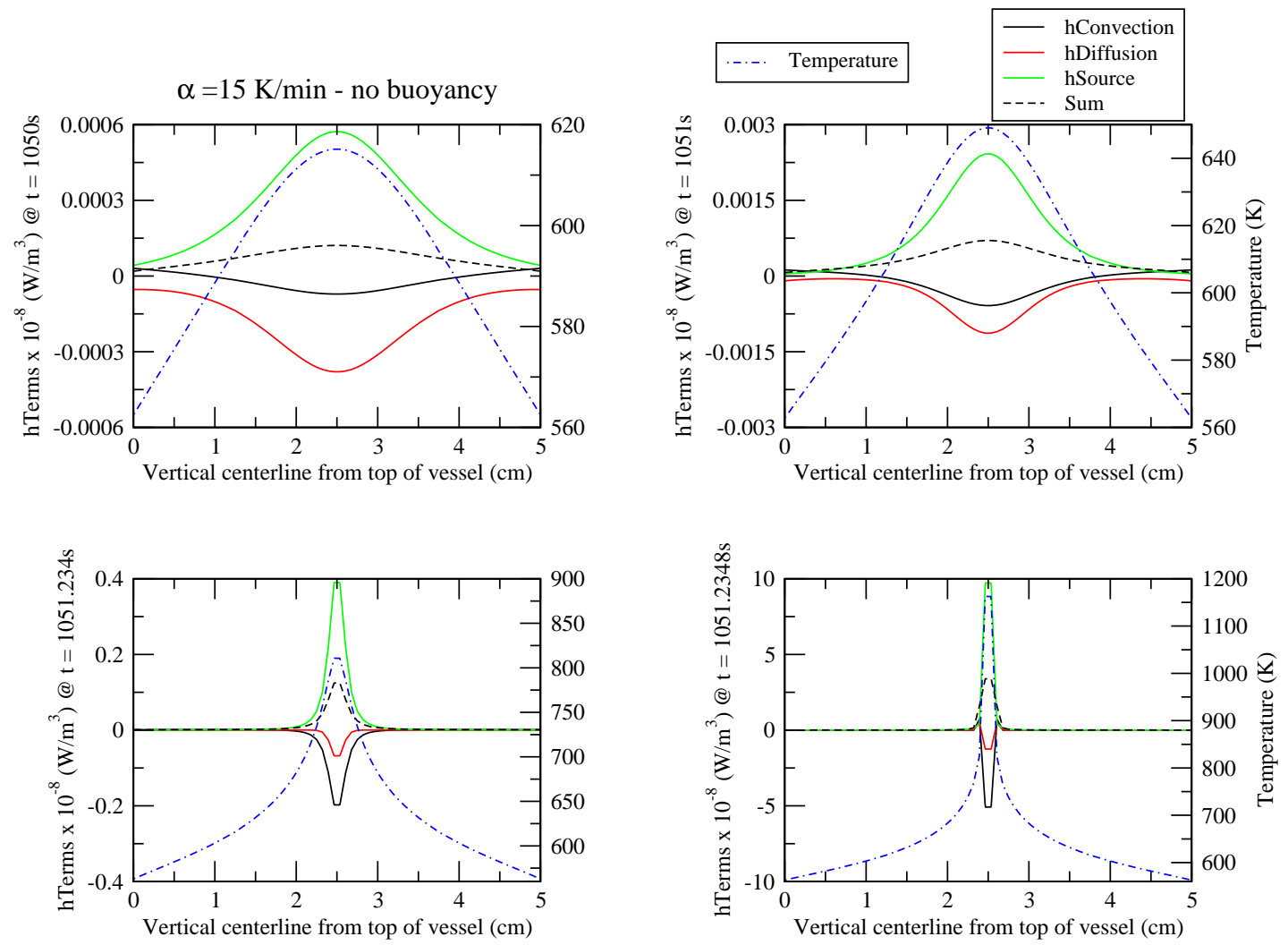

Figure 10: 2-D model simulation results - evolution of ignition event for $\alpha=15 \mathrm{~K} / \mathrm{min}$ applied to a uniformly heated cylindrical reactor neglecting buoyancy: contributions of each term in energy equation and temperature along vertical centerline from top of vessel. Top Left: at t $=1050 \mathrm{~s}-$ early times. Top Right: at $\mathrm{t}=1051 \mathrm{~s}$ - shortly before ignition. Bottom Left: at $\mathrm{t}=1051.234 \mathrm{~s}$ ignition kernel formation. Bottom right: at $\mathrm{t}=1051.2348 \mathrm{~s}$ - ignition kernel structure.

around $937 \mathrm{~s}$, reaction takes place violently with full consumption of the combustible mixture over $5 \mathrm{~ms}$, and equalization with $T_{w}(t)$ after $3 \mathrm{~s}$. The temperature peaks to $\sim 2300 \mathrm{~K}$ during ignition $(\mathrm{t} \sim 1114.73 \mathrm{~s})$. Note that the presence of natural convection expectedly resulted in a delayed ignition event (took $6 \%$ longer to ignite), in line with the observations of Jones [13].

\subsection{Flow analysis $-\alpha=15 \mathrm{~K} / \mathrm{min}$}

Figure 12 shows temperature, velocity and product mass fraction fields together with streamlines and velocity vectors during the initial heating of the reactor for $\alpha=15 \mathrm{~K} / \mathrm{min}$. Until $804 \mathrm{~s}$, the temperature maximum occurs at the wall and the gas is essentially heated uniformly throughout the vessel. The heating of the wall induces a buoyancy flow in which the mixture rises along the walls of the reactor and turns 


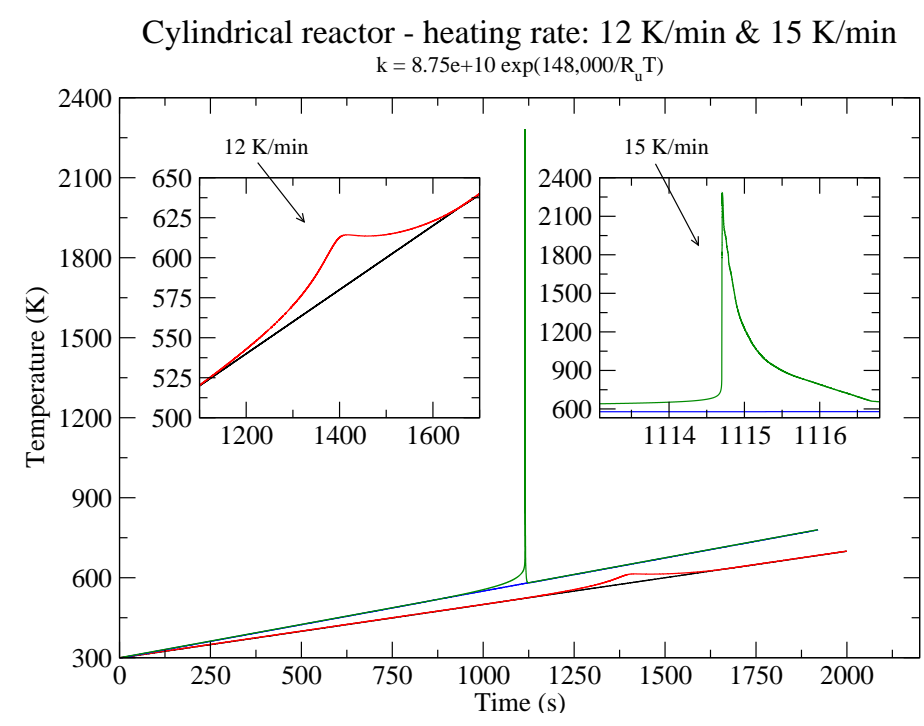

Figure 11: 2-D model simulation results - temperature histories of a uniformly heated cylindrical reactor for $\alpha=12 \mathrm{~K} / \mathrm{min}$ and $15 \mathrm{~K} / \mathrm{min}$.

at the top of the vessel creating two well-defined vortical structures. Competition between the wall's heating and chemical reaction ensues. The chemical heat release rate is higher than the wall's heating rate, diffusive and convective losses combined, which results in the flow being slowed down and reversed: the gas then rises along the center of the reactor, turns as it approaches the top wall, and descends along the side walls (see velocity fields between $t=804 \mathrm{~s}$ and $t=1080 \mathrm{~s}$ in Fig. 12).

As the heating continues, heat addition by chemical reaction increases and the flow is accelerated further to $5 \times 10^{-2} \mathrm{~m} / \mathrm{s}$ at $\mathrm{t}=1114 \mathrm{~s}$. Note that the heat release due to chemistry, together with buoyancy, maintain the hottest gas confined to the top of the reactor (see fields at 1080 and 1114s). As natural convection is more intense, the hot zone moves upwards along the vertical axis, closer to the top of the vessel. Both vortices are also pushed sideways towards the walls. A similar evolution was briefly described in numerical simulations performed for spherical vessels with isothermal boundary conditions [14, 21], and allowing for heat transfer from the reactor's walls to its surroundings characterized by constant Biot number [22]. Consistent with the region where the temperature is highest, and with the flow pattern inside the vessel, the product mass fraction fields show that chemical activity is stronger at the top of the combustion vessel. These fields also reveal the 


\begin{tabular}{|c|c|c|c|}
\hline & Temperature (K) & Velocity $(\mathrm{m} / \mathrm{s})$ & Product (-) \\
\hline $\begin{array}{l}\infty \\
\underset{\infty}{\infty} \\
\| \\
+\infty\end{array}$ & $\begin{array}{l}1167 \\
1040 \\
928 \\
828 \\
-738 \\
659 \\
587 \\
524 \\
467 \\
417\end{array}$ & 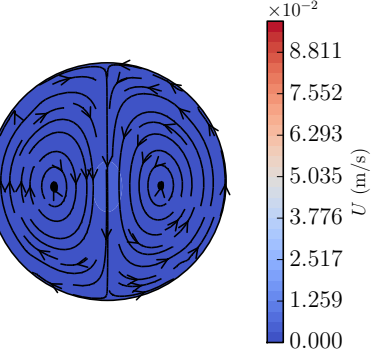 & $\begin{array}{l}0.92 \\
0.79 \\
0.66 \\
0.53 \\
0.40 \\
0.26 \\
0.13 \\
0.00\end{array}$ \\
\hline $\begin{array}{l}\infty \\
\infty \\
\infty \\
0 \\
-1 \\
\| \\
+\end{array}$ & $\begin{array}{l}1167 \\
1040 \\
928 \\
828 \\
738 \\
659 \\
587 \\
524 \\
467 \\
417\end{array}$ & 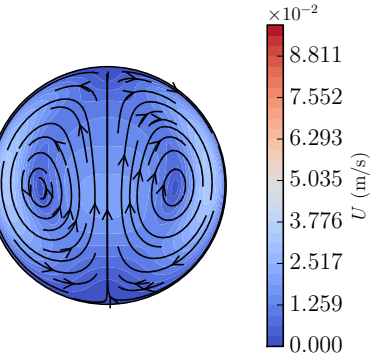 & $\begin{array}{l}0.92 \\
0.79 \\
0.66 \\
0.53 \text { 工्ञ } \\
0.40 \\
0.26 \\
0.13 \\
0.00\end{array}$ \\
\hline 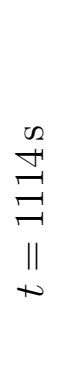 & $\begin{array}{l}1167 \\
1040 \\
928 \\
-828 \\
-738 \\
659 \\
587 \\
524 \\
467 \\
417\end{array}$ & 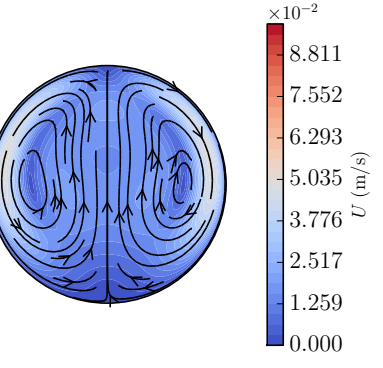 & $\begin{array}{l}0.92 \\
0.79 \\
0.66 \\
0.53 \\
0.40 \\
0.26 \\
0.13 \\
0.00\end{array}$ \\
\hline
\end{tabular}

Figure 12: 2-D model simulation results (fast reaction) - temperature (left), velocity (middle) and product mass fraction (right) fields - early times for $\alpha=15 \mathrm{~K} / \mathrm{min}$ applied to a uniformly heated cylindrical reactor.

extent of mixing that takes place inside the reactor. Both vortices transport hot, partially reacted mixture from the top to the bottom.

Closer to ignition, at $\mathrm{t}=1114.7 \mathrm{~s}$ in Fig. 13, mixing and velocity continue to increase, but as time progresses (specifically over $35 \mathrm{~ms}$ ), a region of localized chemical activity and associated temperature increase appears close to the region where the flow turns. The temperature of the gas in this zone is $243 \mathrm{~K}$ higher than that of the wall (see Fig. 13 at $\mathrm{t}=1114.735 \mathrm{~s}$ ). The main ignition event takes place shortly after, at $\mathrm{t}=1114.7362 \mathrm{~s}$ and brings the temperature of the gas to $\sim 1207 \mathrm{~K}$ over $1.2 \mathrm{~ms}$. The expansion of the gas in the vicinity of the ignition kernel causes the flow to reverse once again, distorting the flow pattern inside the vessel. The 
velocity vectors and streamlines in Fig. 13 at $\mathrm{t}=1114.7362 \mathrm{~s}$ show this clearly. A flame kernel forms at $\mathrm{t}=1114.7375 \mathrm{~s}$ and propagates downwards to the bottom of the reactor fully consuming the mixture in $2.5 \mathrm{~ms}$ (see Fig. 14).

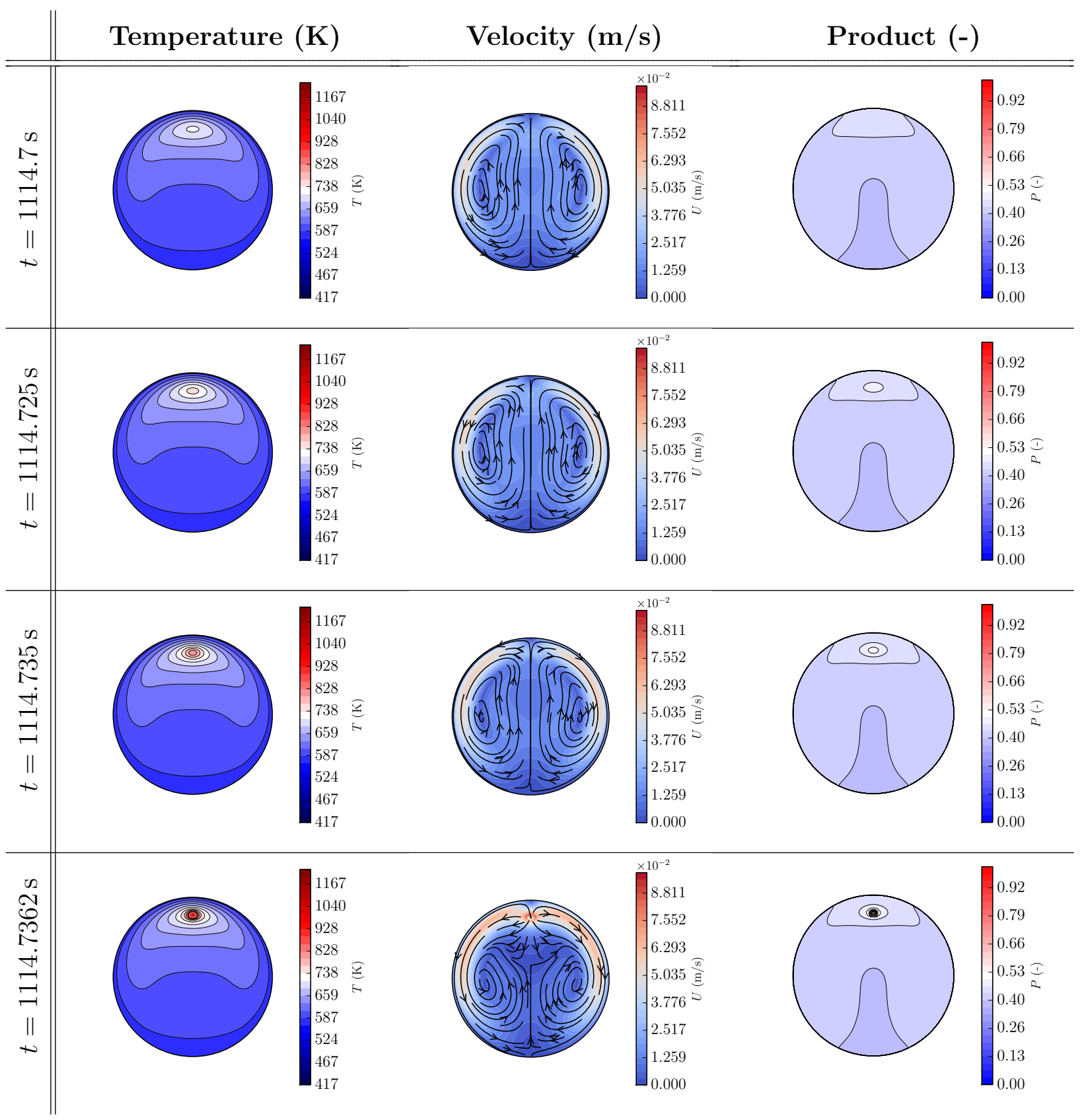

Figure 13: 2-D model simulation results (fast reaction) - temperature (left), velocity (middle) and product mass fraction (right) fields - shortly before ignition and ignition kernel for $\alpha=15 \mathrm{~K} / \mathrm{min}$ applied to a uniformly heated cylindrical reactor. 


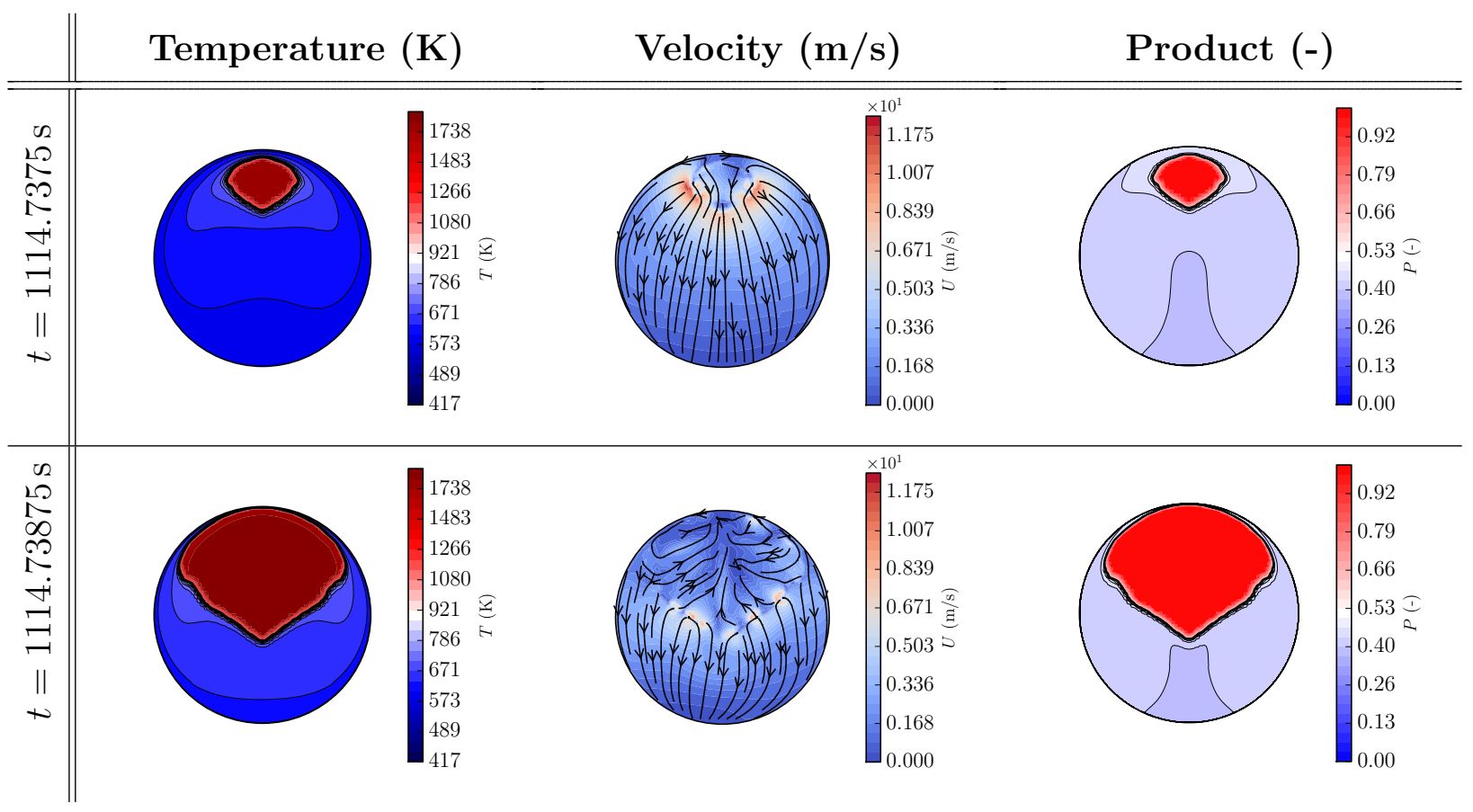

Figure 14: 2-D model simulation results (fast reaction) - temperature (left), velocity (middle) and product mass fraction (right) fields - flame kernel formation and flame propagation for $\alpha=15$ $\mathrm{K} /$ min applied to a uniformly heated cylindrical reactor.

\subsection{Flow analysis $-\alpha=12 \mathrm{~K} / \mathrm{min}$}

At early times, the evolution is similar to that described above for $15 \mathrm{~K} / \mathrm{min}$. This can be observed in Fig. 15 for $\mathrm{t}=1008 \mathrm{~s}$. At $\mathrm{t}=1128 \mathrm{~s}$, flow reversal occurs, and the temperature maximum now lies inside the reactor. The velocities induced in this case are significantly lower than those of the previous case (5 times lower) when comparing similar physical times during the simulation, namely $\mathrm{t}=1200 \mathrm{~s}$ for $12 \mathrm{~K} / \mathrm{min}$ and $\mathrm{t}=1114 \mathrm{~s}$ for $15 \mathrm{~K} / \mathrm{min}$. The mixing and extent of chemical activity is incipient, with imperceptible concentration differences within the reactor (see mass fraction field at $\mathrm{t}=1200 \mathrm{~s})$.

Figure 16 shows that at $12 \mathrm{~K} / \mathrm{min}$ the mixture undergoes very slow consumption rather than an ignition event. At $\mathrm{t}=1344 \mathrm{~s}$ differences between the hottest and coldest region inside the reactor develop evidenced by the closed temperature contours. Velocity continues to increase between $t=1200$ and $1344 \mathrm{~s}$. The peak in velocity, temperature difference between reactor's wall and gas, and product mass fraction difference inside the vessel is reached at $1404 \mathrm{~s}$, with values of $0.039 \mathrm{~m} / \mathrm{s}$, $33 \mathrm{~K}$ and $8.9 \times 10^{-3}$ respectively. The flow structure continues to consist of two 


Temperature $(\mathbf{K}) \quad$ Pelocity $(\mathbf{m} / \mathbf{s}) \quad$ Product (-)

Figure 15: 2-D model simulation results (slow reaction) - temperature (left), velocity (middle) and product mass fraction (right) fields - early times for $\alpha=12 \mathrm{~K} / \mathrm{min}$ applied to a uniformly heated cylindrical reactor.

vortices with the hottest gas confined at the top of the vessel. Although reactant consumption is evident at this heating rate between times $1344 \mathrm{~s}$ and $1464 \mathrm{~s}$, the diffusive time scale is smaller than the chemical heating time scale resulting in no spatial variations in product mass fraction inside the reactor. The weaker natural convection also results in essentially no distortion of the vortices for the duration of the computation. In the bottom half of the reactor, the temperature gradients and buoyancy forces are small resulting in low flow velocities (see $t=1200-1464 \mathrm{~s}$ ). Subsequently, the system relaxes returning to its original configuration with the gas raising along the walls and descending along the vertical centerline of the vessel. The mixture is now fully consumed (product mass fraction $\mathrm{P}=1$ ), see Fig. 16 at 
$\mathrm{t}=1740 \mathrm{~s}$.

Temperature (K)

Figure 16: 2-D model simulation results (slow reaction) - temperature (left), velocity (middle) and product mass fraction (right) fields - slow consumption for $\alpha=12 \mathrm{~K} / \mathrm{min}$ applied to a uniformly heated cylindrical reactor.

8.4. Evolution of temperature, velocity and product mass fraction along vertical centerline

For $15 \mathrm{~K} / \mathrm{min}$, Fig. 17, the temperature profiles show that significant vertical temperature gradients start to develop after $1080 \mathrm{~s}$ (values of $T / T_{w}(t)>1$ ). The 
product mass fraction, $\Lambda$, closely follows the temperature profiles showing a slight departure from unity close to the top of the vessel at 1080 s. Shortly after, the temperature and product mass fraction rise is confined to $0-1 \mathrm{~cm}$, and finally at $1114.7362 \mathrm{~s}$ an ignition kernel forms $\sim 0.5 \mathrm{~cm}$ away from the top wall of the reactor. The velocity profiles show that at early times (up to $804 \mathrm{~s}$ ) the gas travels downwards along the centerline, and reverses between 804-1080 s. Once chemical activity becomes stronger, the heat release, and associated temperature rise accelerates the flow as evidenced by the large velocity increase that takes place between 1114.725 1114.735 s. During ignition, the gas is further accelerated and pushed outwards (see velocity profile at $\mathrm{t}=1114.7362 \mathrm{~s})$.

For $12 \mathrm{~K} / \mathrm{min}$, Fig. 18, the changes are very small along the vertical axis of the vessel. Departures of at most 6 and $2 \%$ in temperature and product mass fraction with respect to their reference values, respectively. The peak in temperature and chemical heat release occurs further away from the top of the vessel $(\sim 1 \mathrm{~cm})$, and no significant acceleration is evident in the velocity profiles during reactant consumption. As chemistry decays, the temperature and mass fraction profiles return to unity, and the gas slowly reverses taking the same configuration it had during the early stages of heating ( see $t=1008 \mathrm{~s}$ and $1740 \mathrm{~s}$ ). Temperature and product mass fraction remain essentially uniform during the entire process.

\subsection{Energy equation analysis}

For $15 \mathrm{~K} / \mathrm{min}$, Fig. 19 at $\mathrm{t}=804 \mathrm{~s}$, the temperature distribution clearly shows the maxima located at the wall. Note that the temperature difference between the wall and the center of the vessel is $0.75 \mathrm{~K}$, which compares very well with the estimate obtained in Section 6. On this ordinate scale $\left(10^{8} \mathrm{~W} / \mathrm{m}^{3}\right)$, all the terms remain in balance. Shortly before ignition $(\mathrm{t}=1114.735 \mathrm{~s})$ the competition between convective and diffusive losses, and chemistry is clear. The increase in temperature is driven by having a higher heat release rate than the rates at which heat is diffused back to the wall and convected inside the reactor. The plot at $\mathrm{t}=1114.7362 \mathrm{~s}$ shows 

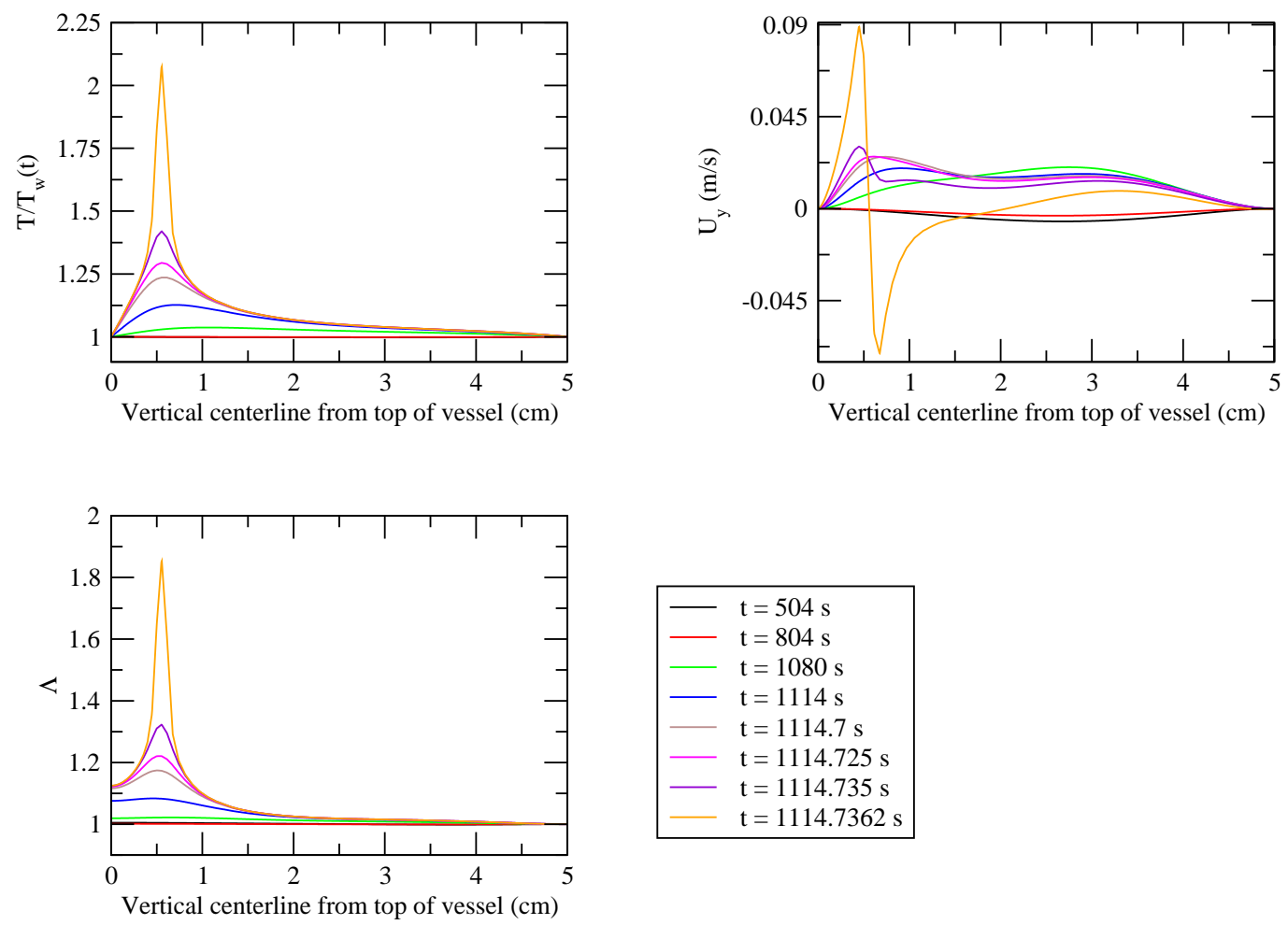

Figure 17: 2-D model simulation results (fast reaction) - normalized temperature $\left(\mathrm{T} / \mathrm{T}_{w}(\mathrm{t})\right)$, vertical velocity $\left(\mathrm{U}_{y}\right)$ and normalized product mass fraction profiles $(\Lambda)$ along vertical centerline of heated vessel during ignition evolution for $\alpha=15 \mathrm{~K} / \mathrm{min}$ applied to a uniformly heated cylindrical reactor.

that ignition occurs close to but slightly away from the wall. The structure of a nascent flame can also be observed. Lastly, at $\mathrm{t}=1114.7375 \mathrm{~s}$, the reaction wave that emanates from the ignition center propagates back towards the top wall and downwards to the bottom consuming the fresh mixture left in the reactor.

For the case with a lower heating rate $(12 \mathrm{~K} / \mathrm{min}$ - see Fig. 20), the energy contributions along the diameter of the reactor have the same order of magnitude $\left(10^{4} \mathrm{~W} / \mathrm{m}^{3}\right)$ during the entire evolution. The competition among the terms is more clearly seen at each location along the vessel centerline. At the wall $(0$ and $5 \mathrm{~cm})$, the balance is kept by diffusion and chemical heat release; further away from the wall, diffusion is counteracted by convection and chemistry. Around the center of the reactor, convection is balanced by diffusion and chemical heat release; in the bottom 

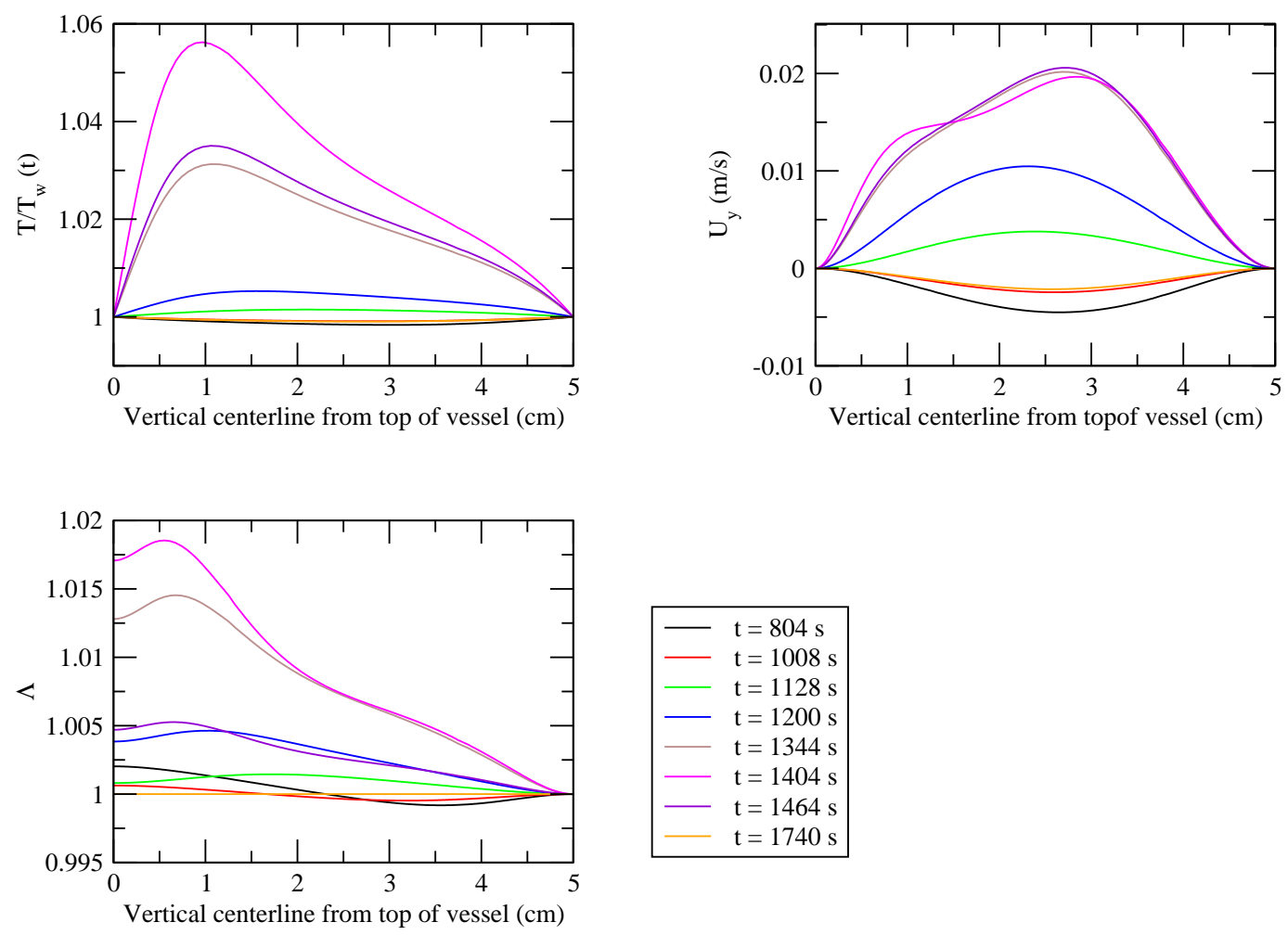

Figure 18: 2-D model simulation results (slow reaction) - normalized temperature $\left(\mathrm{T} / \mathrm{T}_{w}(\mathrm{t})\right.$ ), vertical velocity $\left(\mathrm{U}_{y}\right)$ and normalized product mass fraction profiles $(\Lambda)$ along vertical centerline of heated vessel during ignition evolution for $\alpha=12 \mathrm{~K} / \mathrm{min}$ applied to a uniformly heated cylindrical reactor.

half of the vessel, the convective term decreases to zero and diffusion balances the chemical source term. At $\mathrm{t}=1008 \mathrm{~s}, \Delta T \sim 0.5 \mathrm{~K}$, which is in line with our previous scalings. The distribution of the terms remains essentially unchanged except that at $\mathrm{t}=1404 \mathrm{~s}$ the peak in heat release is more pronounced at $1 \mathrm{~cm}$. The appearance of this peak further away from the top wall is a direct consequence of weaker natural convection. Overall, during the entire consumption process, chemical reaction occurs uniformly within the vessel. This is in stark contrast with the sequence of events seen for $15 \mathrm{~K} / \mathrm{min}$ where localized chemical activity created an ignition center close to the top wall of the reactor. 

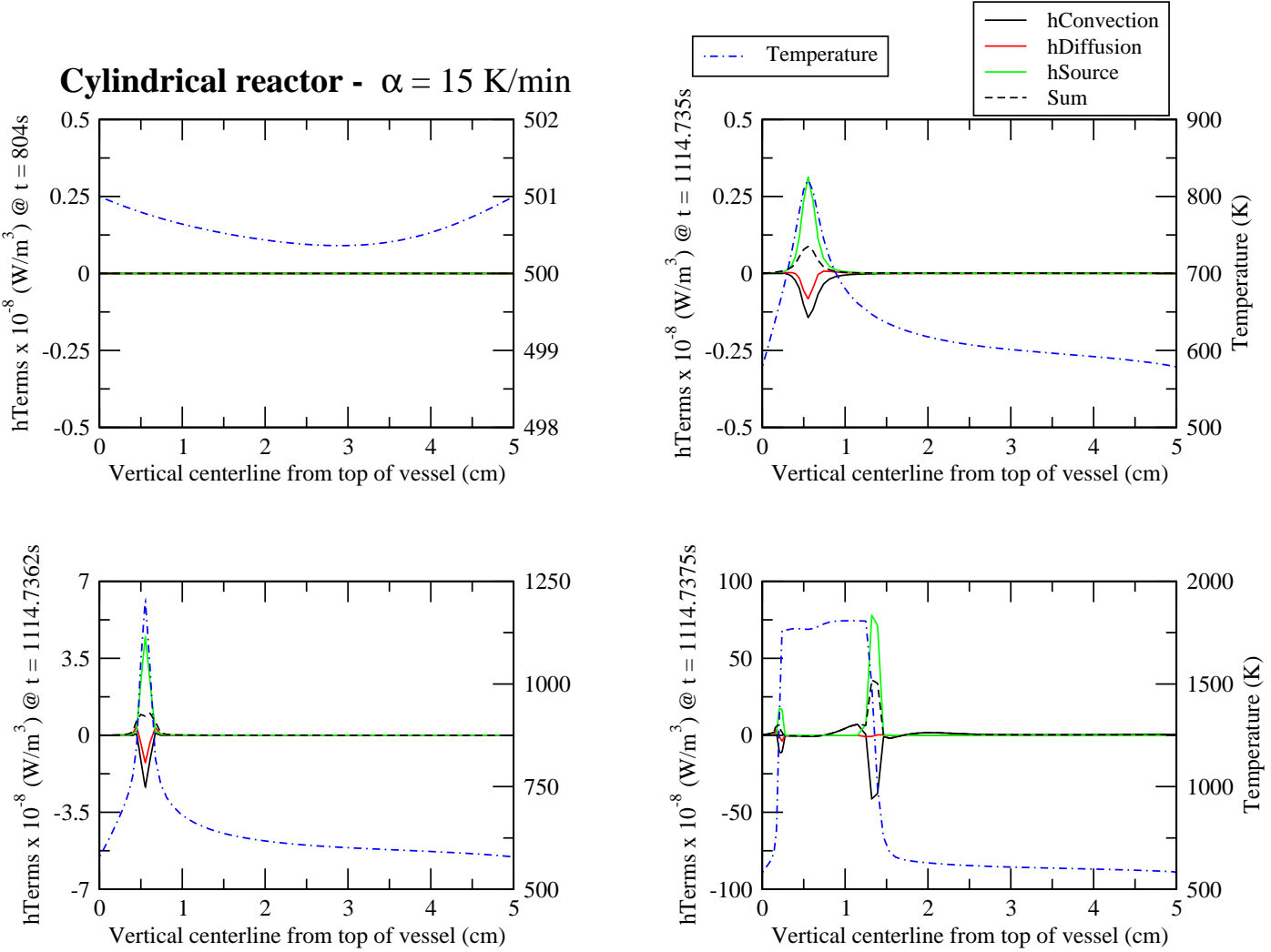

Figure 19: 2-D model simulation results (fast reaction) - evolution of ignition event for $\alpha=15$ $\mathrm{K} / \mathrm{min}$ applied to a uniformly heated cylindrical reactor: contributions of each term in energy equation and temperature along vertical centerline from top of vessel. Top Left: at $\mathrm{t}=804 \mathrm{~s}$ early times. Top Right: at $\mathrm{t}=1114.735 \mathrm{~s}$ - shorly before ignition. Bottom Left: at $\mathrm{t}=1114.7362$ $\mathrm{s}$ - ignition/flame kernel formation. Bottom right: at $\mathrm{t}=1114.7375 \mathrm{~s}$ - early stages of flame propagation.

\section{Non-uniform heating}

The initial and boundary conditions remain unchanged for the most part, except that at both edges of the horizonal centerline of the vessel, a section of $12 \mathrm{~mm}$ is kept at $300 \mathrm{~K}$ for the duration of the simulation (see Fig. 4). Although in reality the windows will not remain at a constant temperature of $300 \mathrm{~K}$ throughout the experiment -which implies infinite heat transfer with its surroundings-, considering the limiting case of having an isothermal boundary condition enhances the important features present in the flowfield. 

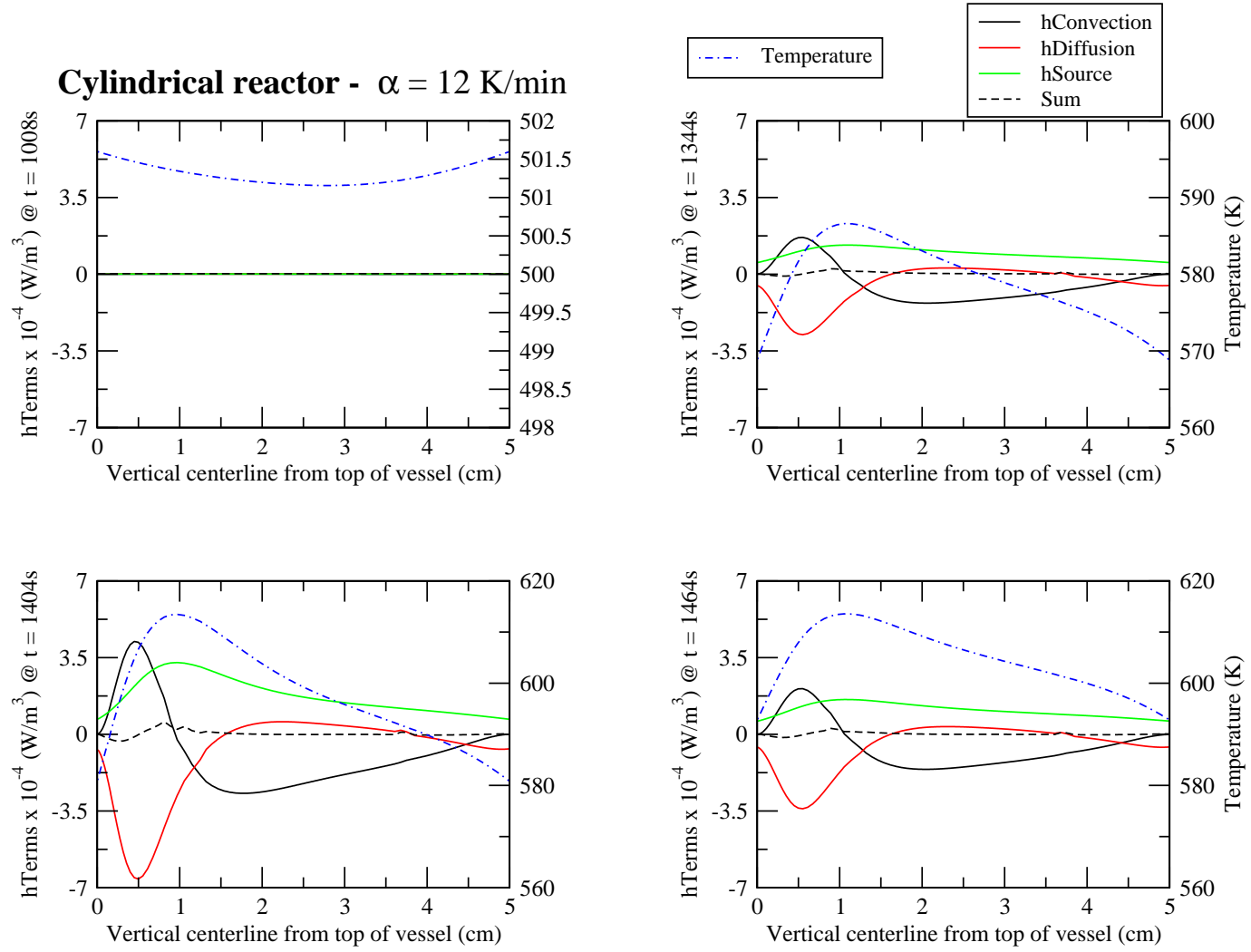

Figure 20: 2-D model simulation results (slow reaction) - evolution of ignition event for $\alpha=12$ $\mathrm{K} / \mathrm{min}$ applied to a uniformly heated cylindrical reactor: contributions of each term in energy equation and temperature along vertical centerline from top of vessel. Top Left: at $\mathrm{t}=1008 \mathrm{~s}-$ early times. Top Right: at $\mathrm{t}=1344 \mathrm{~s}$ - initial slow chemical activity. Bottom Left: at $\mathrm{t}=1404 \mathrm{~s}$ - peak of slow chemical activity. Bottom right: at $\mathrm{t}=1464 \mathrm{~s}$ - decay in slow chemical activity.

\subsection{Time evolution - slow reaction and ignition event}

Both reaction regimes are observed with this configuration, but now the transition occurs at a higher heating rate, between $25 \mathrm{~K} / \mathrm{min}$ and $27 \mathrm{~K} / \mathrm{min}$. Figure 21 shows the temperature histories obtained, and corresponding close-ups of the slow oxidation and ignition events. Full consumption of reactants and equalization with $T_{w}(t)$ occurs over $90 \mathrm{~s}$ and $3 \mathrm{~s}$ respectively. The time to ignition for $\alpha=27 \mathrm{~K} / \mathrm{min}$ is $t \sim 750 \mathrm{~s}$. The combustion products temperature reaches $1900 \mathrm{~K}$ after the ignition event. This peak temperature is lower than in the homogeneous cases due to the cooling of the fluid when in contact with the cold windows. For $\alpha=25 \mathrm{~K} / \mathrm{min}$, slow reaction starts around $t \sim 760 \mathrm{~s}$ which corresponds to a mixture temperature of $\sim 616 \mathrm{~K}$. The departure from the temperature ramp imposed at the walls is very 
weak in this case.

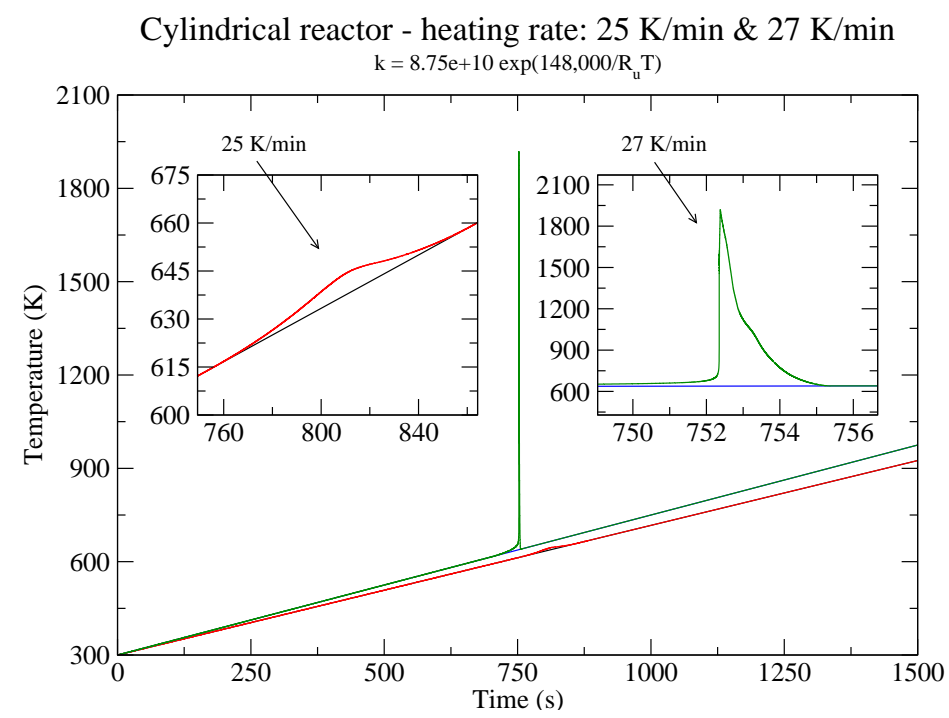

Figure 21: 2-D model simulation results - temperature histories of a non-uniformly heated cylindrical reactor for $\alpha=25 \mathrm{~K} / \mathrm{min}$ and $27 \mathrm{~K} / \mathrm{min}$.

\subsection{Flow analysis $-\alpha=27 \mathrm{~K} / \mathrm{min}$}

The presence of the cold windows results in a very different flow field inside the vessel compared to that observed for the uniform heating case. During early stages of heating (Fig. $22-t=540 \mathrm{~s}$ ), the initial transient is composed of six visible vortices. Four small vortices close to the top and bottom of the reactor induced by the heating of the walls in these regions, and two large vortices in the center. The colder gas next to the windows has the tendency to descend but does not reach the bottom because of the two vortices created by the heating of the walls. The gas rises along the centerline and turns before reaching the top of the vessel as it encounters the two vortices trapped in this region. The temperature field shows the stratification within the combustion vessel: hot gas at the top and bottom, and colder fluid close to the windows. The conversion of fuel into combustion products shows a non-uniform distribution due mostly to the presence of the windows, and to a lesser degree, to the buoyancy flow induced. Chemical activity is highest at the top of the reactor and lowest at the center, consistent with the temperature distribution, where the largest vortices are present and the colder gas mixes within the vessel (see fields at $t=720 \mathrm{~s}$ - Fig. 22) 


Temperature $(\mathbf{K}) \quad$ Velocity $(\mathbf{m} / \mathbf{s}) \quad$ Product (-)

Figure 22: 2-D model simulation results (fast reaction) - temperature (left), velocity (middle) and product mass fraction (right) fields - early times for $\alpha=27 \mathrm{~K} / \mathrm{min}$ applied to a non-uniformly heated cylindrical reactor.

As the heating continues, the flow structure remains unchanged but chemical activity becomes stronger and more localized. The gas begins to expand as a result of heat release and pushes the gas away from this region, causing a slight velocity decrease (see Fig. $23 t=752.342-752.348 \mathrm{~s}$ ). Two milliseconds later, at $t=$ $752.350 \mathrm{~s}$, an ignition kernel forms with the temperature peaking at $1447 \mathrm{~K}$. A flame emanating from the top of the vessel consumes the mixture exactly in the same fashion as in the uniform heating case. The inherent non-uniformities in the flow field just described, could lead to differences in species concentrations and temperature readings of around $35 \%$ (see Fig. 23) . It is thus important for experimentalists to be mindful of these and correct for them. Multi-line laser absortion diagnostics could 
be employed under this kind of experimental configuration [48].

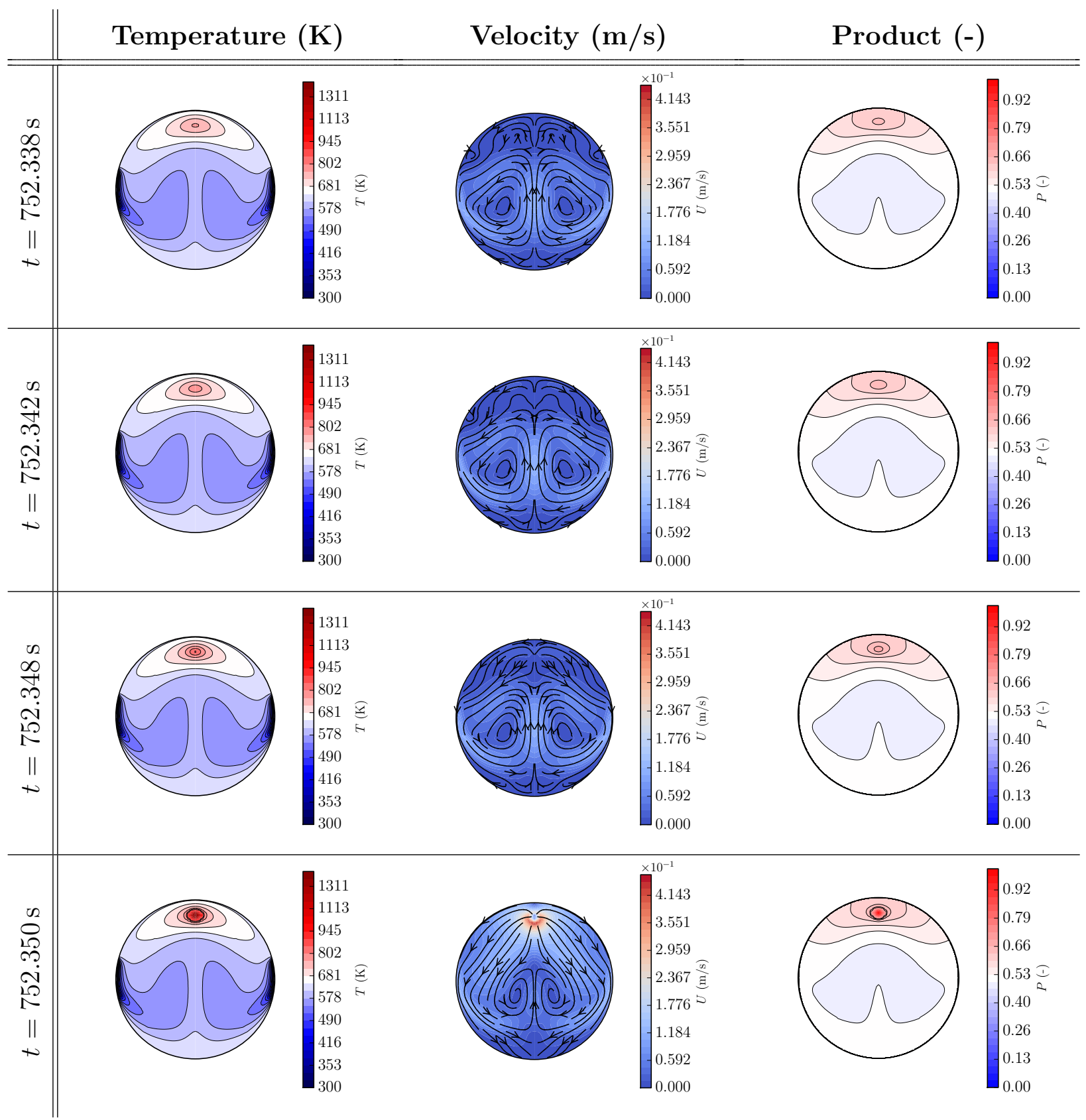

Figure 23: 2-D model simulation results (fast reaction) - temperature (left), velocity (middle) and product mass fraction (right) fields - shortly before ignition and ignition kernel for $\alpha=27 \mathrm{~K} / \mathrm{min}$ applied to a non-uniformly heated cylindrical reactor.

\subsection{Flow analysis $-\alpha=25 \mathrm{~K} / \mathrm{min}$}

For the slow reaction case, the behavior during early times is the same as for $\alpha=$ $27 \mathrm{~K} / \mathrm{min}$. At later times, even though most of the chemical activity is still confined 
to the top of the vessel, the differences between highest and lowest concentration of combustion products are at most $\sim 13 \%$. Full depletion of the fuel takes place with a very weak temperature rise $(10 \mathrm{~K}$ above the prescribed heating ramp at the walls), and more or less "uniformly" within the reactor without the presence of strong localized chemical activity nor a flame. The colder unreacted gas present in the center is transported and fed to the top of the combustion vessel by the two largest vortices present in the flow field. In contrast with the $27 \mathrm{~K} / \mathrm{min}$ case, no perceptible velocity decrease is observed because of the absence of an ignition kernel and associated expansion of the gas.

\subsection{Energy equation analysis}

In the $\alpha=27 \mathrm{~K} / \mathrm{min}$ case, the behavior is very similar to that described in section 8 except for the lower temperature present in the center of the vessel where the cold windows are located. The temperature minimum is $75 \mathrm{~K}$ less than the prescribed ramp at the wall (see Fig 25 at $t=612 \mathrm{~s}$ ). At $t=752.348 \mathrm{~s}$, a region of localized chemical activity is present roughly $0.5 \mathrm{~cm}$ away from the wall. Two milliseconds later, at $t=752.350 \mathrm{~s}$, an ignition kernel forms and an incipient flame propagates towards the top and bottom walls. The double structure present is evidence of the radial expansion of the reaction front. The final plot in Fig. 25, $t=752.358 \mathrm{~s}$, shows the flame propagating downwards with the temperature peaking at $1700 \mathrm{~K}$.

Lastly, the energy equation analysis for $\alpha=25 \mathrm{~K} / \mathrm{min}$ shows a very different evolution from that shown for the uniform heating case. A stratified flow structure is seen: (i) almost quiescent mixture present up to $0.5 \mathrm{~cm}$ away from the top wall (convective losses are negligible), (ii) colder gas that is fed from the center of the vessel to the top between $1-2 \mathrm{~cm}$, (iii) two large vortical structures in the $2-4.5 \mathrm{~cm}$ region, and (iv) from $4.5-5 \mathrm{~cm}$, a smaller zone with combustible mixture at "rest" at the bottom of the vessel. From $1-4.5 \mathrm{~cm}$ the balance is maintained by diffusion and convection with very small contributions due to chemistry. Close to the walls, diffusion balances the chemical source term with the temperature peaking to $650 \mathrm{~K}$ at $t=816 \mathrm{~s}$. Finally, at $t=840 \mathrm{~s}$, all fuel is depleted and the temperature maximum 


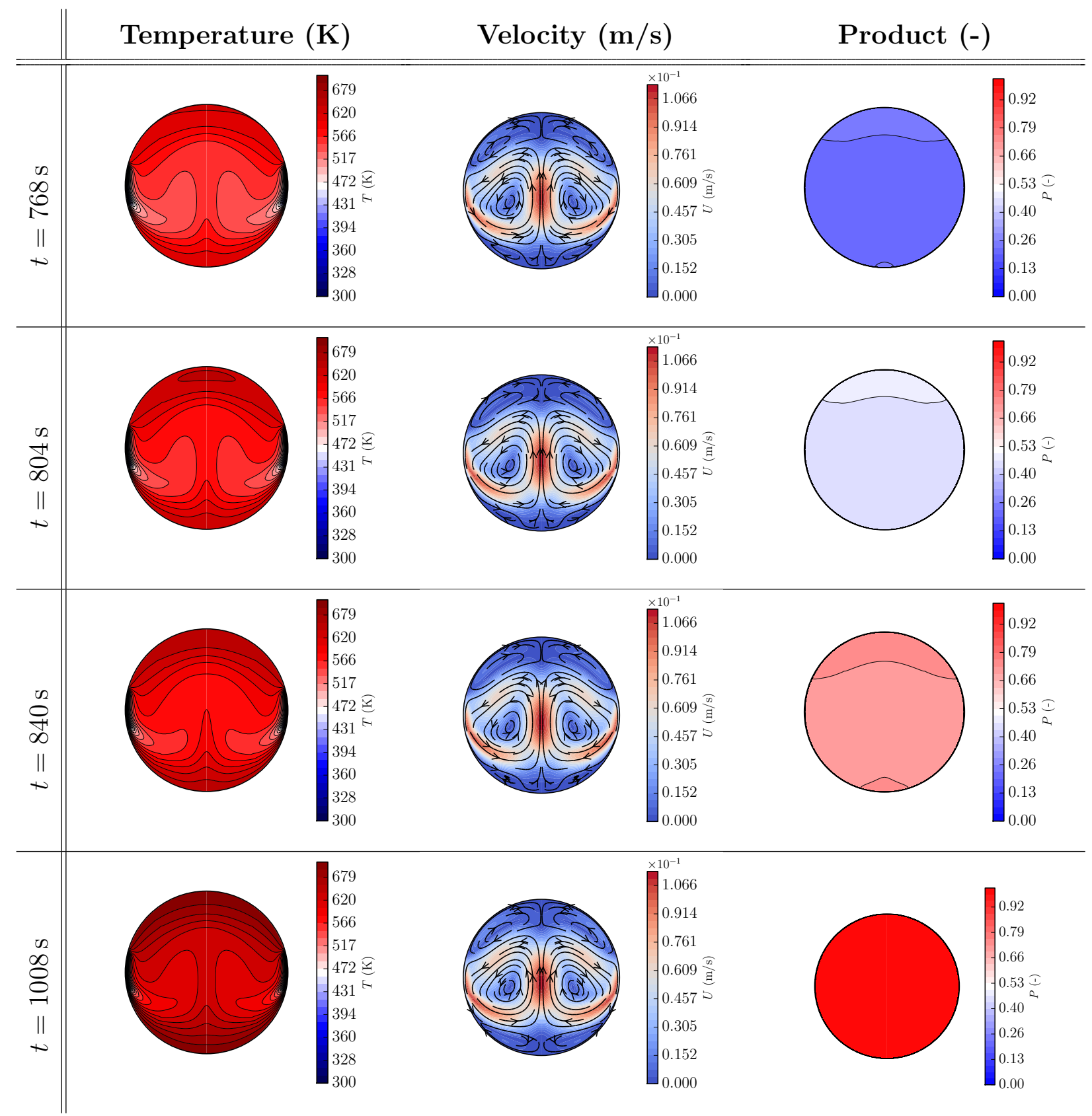

Figure 24: 2-D model simulation results (slow reaction) - temperature (left), velocity (middle) and product mass fraction (right) fields - slow consumption for $\alpha=25 \mathrm{~K} / \mathrm{min}$ applied to a nonuniformly heated cylindrical reactor.

returns to the wall as the mixture continues to be heated. 

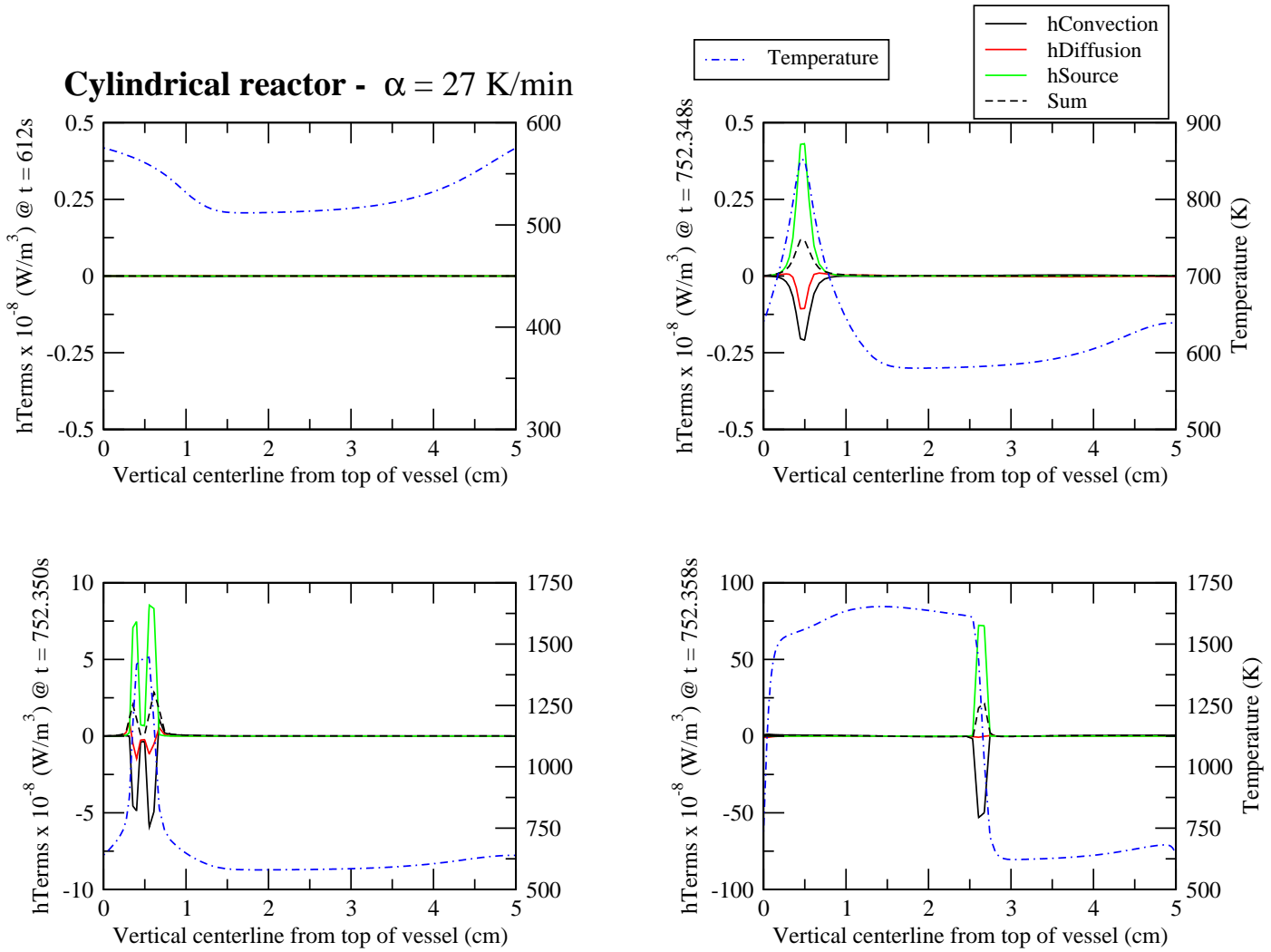

Figure 25: 2-D model simulation results (fast reaction) - evolution of ignition event for $\alpha=27$ $\mathrm{K} / \mathrm{min}$ applied to a non-uniformly heated cylindrical reactor: contributions of each term in energy equation and temperature along vertical centerline from top of vessel. Top Left: at $\mathrm{t}=612 \mathrm{~s}-$ early times. Top Right: at $\mathrm{t}=752.348 \mathrm{~s}$ - shorly before ignition. Bottom Left: at $\mathrm{t}=752.35 \mathrm{~s}-$ ignition/flame kernel formation. Bottom right: at $\mathrm{t}=752.358 \mathrm{~s}$ - early stages of flame propagation.

\section{Conclusion}

A numerical study of a cylindrical vessel undergoing uniform and non-uniform heating was performed using simplified kinetics. The methodology used to develop one-step chemistry capable of describing the experimentally observed transition from slow to fast reaction regimes was outlined in detail. A parametric study using a constant volume zero-dimensional model was performed to provide transition maps due to changes in heating rate, initial pressure and composition. Two-dimensional simulations focused on the description of the fluid mechanics, and the competition between convective and diffusive losses, and chemical heat release inside the reactor as the heating rate was varied in a uniformly and non-uniformly heated reactor. A time scale analysis based on simple scalings of the governing equations elucidated 

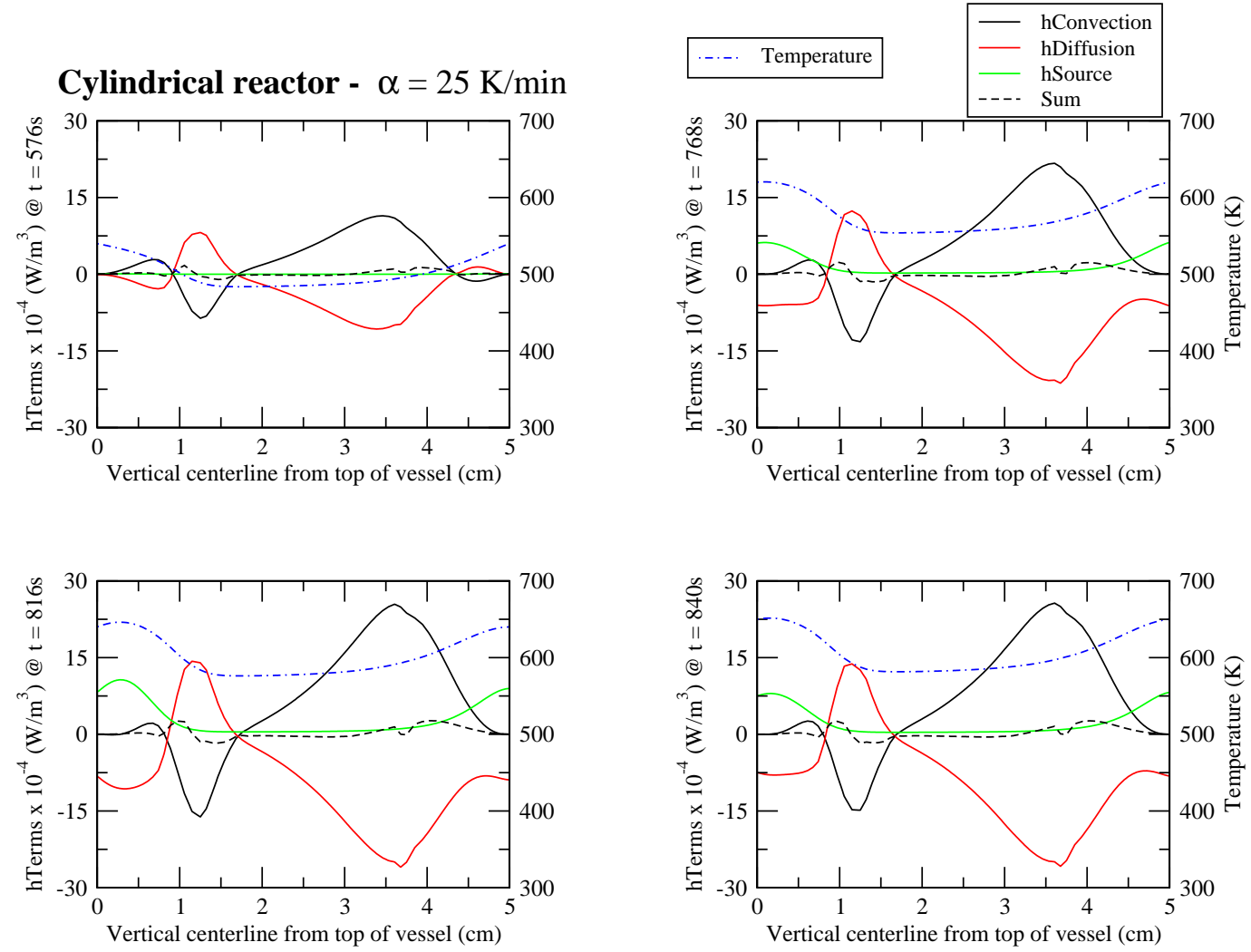

Figure 26: 2-D model simulation results (slow reaction) - evolution of ignition event for $\alpha=25$ $\mathrm{K} / \mathrm{min}$ applied to a non-uniformly heated cylindrical reactor: contributions of each term in energy equation and temperature along vertical centerline from top of vessel. Top Left: at $t=576 \mathrm{~s}$ early times. Top Right: at $\mathrm{t}=768 \mathrm{~s}-$ initial slow chemical activity. Bottom Left: at $\mathrm{t}=816 \mathrm{~s}$ peak of slow chemical activity. Bottom right: at $\mathrm{t}=840 \mathrm{~s}$ - decay in slow chemical activity.

the competition of the different physical processes as a function of temperature; for the cases considered here the crossover between the diffusive and chemical time scales accurately marked the ignition boundary.

To assess the influence of natural convection on the ignition evolution, a buoyancyfree case was used as a reference. In good agreement with previous observations (using spherical reactors and isothermal boundary conditions), natural convection was found to delay ignition. Regarding the ignition location, natural convection moves the ignition kernel from the center towards the upper half of the reactor, depending upon the strength of the buoyancy flow induced in the vessel.

During uniform heating, a drastic change in the reaction behavior occurred when the heating rate was increased from 12 to $15 \mathrm{~K} / \mathrm{min}$. From having slow consumption of 
the mixture at $12 \mathrm{~K} / \mathrm{min}$ to an ignition event at $15 \mathrm{~K} / \mathrm{min}$. At early times, the flow is composed of two main vortical structures for both heating rates. Once significant heat is generated inside the vessel the flow reverses. This flow reversal that appears to be a typical feature of these systems had not been described in previous work. It results from the competition between wall heating and heat release during the early stages of the process. During non-uniform heating, the transition occurred when the rate was increased from 25 to $27 \mathrm{~K} / \mathrm{min}$. At both heating rates, the flow field is composed of two large vortices in the center of the vessel, and two sets of smaller vortices trapped at the top and bottom of the reactor. Uniform and non-uniform heating cases ignited at comparable times and locations. Our results emphasize the importance of the flow pattern developed by the interaction between the vessel containing the reactive mixture, and natural convection in creating zones where the critical conditions for ignition are met (i.e. top of the vessel). This observation is not unique to this configuration, ignition time scale or nature of the flow, but has been discussed in detail recently, in the context of thermal ignition, for different geometries (e.g. transiently heated concentrated surfaces and moving heated particles) $[4,5,6,7,8,9]$.

Acknowledgements The simulations shown and writing of this manuscript were carried out while JM-G was supported by the Natural Sciences and Engineering Research Council of Canada (NSERC) Postdoctoral Fellowship Program. RM was supported by a start-up package from the Center of Combustion and Energy and Tsinghua University and the 1000 Talents of China plan. This work used the Extreme Science and Engineering Discovery Environment (XSEDE), which is supported by the National Science Foundation grant number ACI-1548562. 


\section{References}

[1] P.A. Boettcher, R. Mével, V. Thomas and J.E. Shepherd. Fuel, 96 (2012) 392403.

[2] Boettcher, P. A.. Thermal Ignition. Ph.D Thesis. California Institute of Technology, 2012.

[3] P.A. Boettcher, S.K. Menon, B.L. Ventura, G. Blanquart and J.E. Shepherd. J. Fluid Mech., 735 (2013) 176-202.

[4] J. Melguizo-Gavilanes, L. Boeck, R. Mével and J. E. Shepherd. Int. J. Hydrogen Energ., 42 (2017) 7393-7403.

[5] J. Melguizo-Gavilanes, A. Nové-Josserand, S. Coronel, R. Mével and J. E. Shepherd. Combust. Sci. Technol., 188 (2016) 2060-2076.

[6] S.A. Coronel, J. Melguizo-Gavilanes, R. Mével, and J. E. Shepherd. Combust. Flame, 192 (2018) 495-506.

[7] J. Melguizo-Gavilanes, S. Coronel, R. Mével and J. E. Shepherd. Int. J. Hydrogen Energ., 42 (2017) 7380-7392.

[8] J. Melguizo-Gavilanes, R. Mével, S. Coronel, and J. E. Shepherd. P. Combust. Inst., 36 (2017) 1155-1163.

[9] S. Jones, J. Melguizo-Gavilanes, J. E. Shepherd. P. Combust. Inst., doi: 10.1016/j.proci.2018.07.046

[10] J. Melguizo-Gavilanes, P. A. Boettcher, A. Gagliardi, V.L. Thomas and R. Mével. 9th US National Combustion Meeting. Cincinnati, Ohio May 17-20 (2015)

[11] N. N. Semenov. Progress of Physical Science, 23 (1940).

[12] D. A. Frank-Kamenetskii. Diffusion and heat exchange in chemical kinetics, Princeton University Press, Princeton, NJ, 1955. 
[13] D. R. Jones. Int. J. Heat Mass Transfer, 16 (1973) 157-167.

[14] T.-Y. Liu, A. N. Campbell, S. S. S. Cardoso and A. N. Hayhurst. Phys. Chem. Chem. Phys., 10 (2008) 5521-5530.

[15] P. L. Chambre. J. Chem. Phys., 20 (1952) 1795-1797.

[16] J. R. Parks. J. Chem. Phys., 34 (1961) 46-50.

[17] A. G. Merzhanov. Combust. Flame., 10 (1966) 341-348.

[18] T. Boddington, P. Gray and D. I. Harvey. Philos. Trans. R. Soc. London. Ser. A, 270 (1971) 467-506.

[19] T. Boddington, C.-G. Feng and P. Gray. Philos. Trans. R. Soc. London. Ser. A, 390 (1983) 247-264.

[20] T. Boddington, C.-G. Feng and P. Gray. Philos. Trans. R. Soc. London. Ser. A, 392 (1984) 301-322.

[21] T.-Y. Liu, A. N. Campbell, A. N. Hayhurst and S. S. S. Cardoso. Combust. Flame, 157 (2010) 230-239.

[22] A. N. Campbell. Phys. Chem. Chem. Phys., 17 (2015) 16894-16906.

[23] I. Iglesias, D. Moreno-Boza, A. L. Sánchez, A. Liñán, F. A. Williams. Int. J. Heat Mass Transfer, 115 (2017) 1042-1053.

[24] I. Glassman. Combustion. Academic Press, San Diego, 4th edition, 2008.

[25] S. P. M. Bane, J. L. Ziegler and J. E. Shepherd. Development of one-step chemistry models for flame and ignition simulation. GALCIT Report GALTCITFM:2010.002, 2010.

[26] F. M. White. Heat Transfer. Addison-Wesley, Boston, MA, 1984.

[27] H. P. Ramirez, K. Hadj-Ali, P. Dievart, G. Dayma, C. Togbe, G. Moreac and P. Dagaut. Proc. Combust. Inst., 33 (2011) 375-382. 
[28] R. Mével, K. Chatelain, P. A. Boettcher and J. E. Shepherd. Fuel, 126 (2014) 282-293.

[29] D. Goodwin. Cantera: object-oriented software for reacting flows. Technical Report, California Institute of Technology, 2005.

[30] P. A. Boettcher, V. L. Thomas and R. Mével. Western States Section of the Combustion Institute. California Institute of Technology, March 23-25 (2014)

[31] W. Sutherland. The London, Edinburgh, and Dublin Philosophical Magazine and Journal of Science, 36 (1893) 507-531.

[32] B. E. Poling, J. M. Prausnitz, J. P. O'connell, et al. The properties of gases and liquids. volume 5, Mcgraw-hill New York, 2001.

[33] A. Burcat and B. Ruscic Third millenium ideal gas and condensed phase thermochemical database for combustion with updates from active thermochemical tables, Argonne National Laboratory Argonne, IL, 2005.

[34] H. G. Weller, G. Tabor, H. Jasak, and C. Fureby. Comput. Phys., 12 (1998) $620-631$

[35] Y. Saad, Iterative Methods for Sparse Linear Systems, SIAM, Philadelphia PA, USA, 2003, p.528.

[36] I. Demirdzic, Z. Lilek, M. Péric, Int. J. Numer. Meth. Fl., 16 (1993) 1029-1050

[37] E. S. Oran, J. P. Boris, Numerical Simulation of Reactive Flow, Cambridge University Press, New York NY, USA, 2001, p.529.

[38] F. Zhang, H. Bonart, T. Zirwes, P. Habisreuther, H. Bockhorn, N. Zarzalis, In book: High Performance Computing in Science and Engineering '14, Springer International Publishing, 2015, 221-236

[39] Bonart, H. Implementation and Validation of a Solver for Direct Numerical Simulations of Turbulent Reacting Flows in OpenFOAM. Bachelor Thesis. Karlsruhe Institute of Technology, 2012. 
[40] O. A. Marzouk, E. D. Huckaby. Eng. App. Comput. Fluid Mech., 4:3 (2010) 331-356

[41] A. Cuoci, A. Frassoldati, T. Faravelli, E. Ranzi. Combust. Flame, 160:5 (2013) $870-886$

[42] A. Cuoci, T. Frassoldati, T. Faravelli, E. Ranzi. Energ. Fuel, 27:12 (2013) $7730-7753$

[43] M. Maestri, A. Cuoci. Chem. Eng. Sci., 96 (2013) 106-117

[44] V. Vuorinen, A. Wehrfritz, C. Duwig, B. J. Boersma. Fuel, 130:8 (2014) 241-250

[45] O. Kaario, V. Vuorinen, T. Hulkkonen, K. Keskinen, M. Nuutinen, M. Larmi, F. Tanner. Atomization Sprays, 23:4 (2013) 297-325

[46] J. F. Clarke, D. R. Kassoy, N. Riley. Proc. R. Soc. A, 393:1805 (1984) 309-329

[47] J. F. Clarke, D. R. Kassoy, N. Riley. Proc. R. Soc. A, 393:1805 (1984) 331-351

[48] X. Liu, Line-of-sight absorption of $\mathrm{H}_{2} \mathrm{O}$ vapor: gas temperature sensing in uniform and nonuniform flows. Ph.D Thesis. Stanford University, 2006. 